\title{
The zebrafish as a promising tool for modeling human brain disorders: A review based upon an IBNS Symposium
}

\author{
Soaleha Shams ${ }^{1}$, Jason Rihel², Jose G. Ortiz ${ }^{3}$, and Robert Gerlai ${ }^{1,4 *}$
}

\footnotetext{
${ }^{1}$ Department of Cell and System Biology, University of Toronto, Toronto, Canada ${ }^{2}$ Department of Cell \& Developmental Biology, University College London, London UK ${ }^{3}$ Department of Pharmacology and Toxicology, School of Medicine, University of Puerto Rico - Medical Sciences Campus, San Juan, Puerto Rico

${ }^{4}$ Department of Psychology, University of Toronto Mississauga, Mississauga, Canada
}

*Correspondence may be addressed to:

Robert Gerlai, Ph.D.

Department of Psychology, Rm CCT4004

University of Toronto Mississauga

3359 Mississauga Road, Mississauga

Ontario, CANADA, L5L 1 C6

office: (905)569-4255

lab: (905)569-4257

fax: (905)569-4326

e-mail: robert_gerlai@yahoo.com 


\begin{abstract}
The zebrafish represents an excellent compromise between system complexity and practical simplicity, features that make it useful for modeling and mechanistic analysis of complex brain disorders. Also promising are screens for psychoactive drugs with effects on larval and adult zebrafish behavior. This review, based upon a recent symposium held at the 2016 IBNS Congress, provides different perspectives on how the zebrafish may be utilized to advance research into human central nervous system disorders. It starts with a discussion on an important bottleneck in zebrafish research, measuring the behavior of this species (specifically shoaling), and continues with examples on research on autism spectrum disorder in larval zebrafish, on screening natural products for compounds with psychoactive properties in adult zebrafish, and on the development of a zebrafish model of fetal alcohol spectrum disorders. By providing information on a broad spectrum of brain disorders, experimental methods, and scientific approaches using both larval and adult zebrafish, the review is intended to showcase this underutilized laboratory species for behavioral neuroscience and psychopharmacology research.
\end{abstract}

Key words: alcoholism, anxiety, autism, epilepsy, fetal alcohol spectrum disorders, psychopharmacology, social behavior, zebrafish 


\section{Introduction}

Although a relative newcomer compared to classical biomedical laboratory research species including the rat or the mouse, the zebrafish is rapidly gaining popularity in a variety of disciplines of biology, from behavioral neuroscience (Kalueff et al., 2014; Sison et al., 2006) to genetics (Patton \& Zon, 2001). The first zebrafish study, according to ISI Web of Science was published in 1954 (Battle \& Mcleese, 1954). The very first study on record in the PubMed database that used zebrafish with a biomedical focus was published in 1957 (Hisaoka \& Hopper, 1957), and for the next three decades, the number of publications using zebrafish remained low but increasing steadily from a couple of papers to a handful of papers per year. However, since the early nineties, this increase accelerated, jumping from tens of papers to nearly 3000 yearly publications on zebrafish by 2016. Notably, although still orders of magnitude fewer than the number papers on the mouse or the rat, the increase of the number of publications on zebrafish significantly outpaced those on the latter two species (Kalueff et al., 2014).

The reason for the increasing popularity of the zebrafish is that it represents an excellent compromise between system complexity and practical simplicity. It is a vertebrate complex enough to allow sufficient translational relevance (Gerlai, 2014). Most importantly, the nucleotide sequence of its genes has been found to be similar to that of human homologs, often reaching or exceeding $70 \%$ homology (Howe et al., 2013). This high degree of genetic conservation strongly suggests that when genes are found to serve specific functions in zebrafish, similar genes will be involved in the same function in humans. Screening a large number of mutant zebrafish, however, is a lot simpler, and most importantly, a substantially cheaper exercise than doing the same with 
laboratory mammals such as the mouse or the rat. For example, several studies utilize larval zebrafish, i.e. zebrafish that are about 5-10 post-fertilization day old. At this size (around $1 \mathrm{~mm}$ total length) zebrafish is already freely swimming, and exhibits a fairly complex behavioral repertoire and responds to a variety of stimuli of all possible modalities. At this size, the behavioral responses may be quantified using 96 well plates. Numerous commercially available zebrafish movement analysis and stimulus delivery systems have been developed which utilize the 96 well plate set up and thus can measure a large number of fish very quickly. Most behavioral paradigms require no more than 10 min of session length, thus in an 8-hour day, one can obtain detailed video-tracking-based movement analysis data on over 4600 fish, just using a single 96 well plate system. This throughput is about two orders of magnitude faster than what is usually achieved in rodent behavioral screening studies (Gerlai, 2014b; 2010; 2002). Adult zebrafish require more space than larval ones do, but usually even adult fish can be tested much faster than rodents. Numerous behavioral paradigms have been developed for adult zebrafish that allow one to screen hundreds of fish per day even with a single behavioral set up (Gerlai, 2014b; 2010). Also, notably, the zebrafish represents good reduction and refinement from an animal ethics perspective, as it is a simpler vertebrate compared to mammalian laboratory species. Last, the zebrafish is a vertebrate, and thus is substantially more complex and evolutionarily closer to humans than drosophila or the nematode, two of the most frequently employed non-vertebrate model organisms in genetics and neuroscience. In summary, the zebrafish appears to strike an optimal compromise between system complexity and practical simplicity, sophistication and test throughput, and as a result has become one of the primary tools for forward as well as reverse genetic studies 
(Amsterdam and Hopkins, 2006; Chen and Ekker, 2004; Lekven et al., 2000; Patton and Zon, 2001). These studies focused on phenotypes relevant for developmental biology. More recently, however, the zebrafish has been rediscovered for many other domains of investigations, behavioral neuroscience and psychopharmacology being among them (Stewart et al, 2014; Kalueff et al., 2014; Gerlai, 2012).

The zebrafish has been found to possess evolutionarily conserved features at many levels of its biological organization, not just at the level of genetics. For example, it has a typical vertebrate brain that shares its basic neuroanatomical layout with that of other vertebrate species (Tropepe and Sive, 2003), and its neurotransmitter systems are also similar to those of mammals (Chatterjee and Gerlai, 2009). For the latter reason, and not surprisingly, drugs developed for human clinical applications, or for other mammalian species, have often been found efficacious in zebrafish acting through similar if not identical receptors and biochemical mechanisms compared to those of mammals (Kalueff et al., 2014; Levin et al., 2015). For these reasons, the zebrafish has been suggested as an appropriate laboratory tool for the screening of not only mutations but also drugs and compounds. Given the huge number of compounds developed in pharmaceutical research by companies and academic laboratories alike, the need for an efficient screening tool is clear.

This need is further emphasized by the staggering number of human brain disorders for which pharmacotherapy has been insufficient or unavailable (e.g. Klafki et al., 2006; Poewe, 2009; Shelton et al., 2010). Furthermore, the brain is perhaps the most complex organ, and thus the mechanisms of its dysfunctions are often not well understood. In short, the brain is considered one of the final frontiers of biology, and brain disorders 
continue to represent a substantial unmet medical need. To alleviate these problems and to meet this need, animal models with sufficient translational relevance have been suggested for a broad range of human brain disorders and/or for neurobiological mechanisms suspected to be involved in such disorders (Stewart et al., 2014, 2015a; Kalueff et al., 2014; Gerlai, 2010, 2012). The zebrafish may be not only a translationally relevant tool for such studies, but it may also represent one of the most efficient and cheapest approaches. It is a small (4 cm long) animal that can be housed in large numbers cost effectively. It is also highly prolific with a single adult female being able to produce 2-300 eggs 2-3 times per week. Zebrafish phenotyping can be highly automated, which not only allows for repeatable measurements in many animals, but also enables longerterm behavioral observations that may identify phenotypes that may be missed in shortterm observations. Although there are many similarities between the zebrafish and mammalian species, given that the zebrafish is evolutionarily older, its brain is substantially less complex than the mammalian brain, a feature that has been argued to enable one to discover the more fundamental, core, and thus most important mechanisms relevant to the biological phenomenon, or disease, under study (Gerlai, 2014). Thus, the zebrafish should be an excellent drug screening tool. Screening for psychoactive or other brain function altering compounds using the zebrafish, however, may be hindered by numerous factors. First and foremost, appropriate screening methods such as specific behavioral test paradigms are often unavailable for the zebrafish, a species that is a relative newcomer in psychopharmacology. Furthermore, information about drug absorption, distribution, metabolism and excretion (ADME) of compounds is often unavailable in either larval or adult zebrafish. 
This review presents diverse aspects of this novel but rapidly evolving field, zebrafish neuropsychopharmacology. Although this review is far from comprehensive, its topics are chosen to cover a broad spectrum of questions and areas of investigation with zebrafish. It is a sampling of fairly different research areas and methods as well as disease foci, including autism spectrum disorders (ASDs), anxiety, and embryonic alcohol exposure induced problems. The review covers questions related to designing behavioral paradigms for testing drug effects and identifying potentially interesting efficacious compounds and also present examples on how to combine methods and approaches of psychology, ethology, pharmacology, genetics and neurobiology. In summary, this review attempts to showcase the zebrafish as a promising laboratory research organism for psychopharmacology, neurobiology and behavior genetics, while pointing out both the advantages and the current shortcomings of this species in empirical research.

\section{Social behavior of the zebrafish: Why and how should we measure it?}

The relative paucity of appropriate behavioral test paradigms and our limited understanding of the behavior of the zebrafish (Sison et al., 2006) represent important bottlenecks in behavioral genetic, behavioral neuroscience and psychopharmacological analysis of the zebrafish. Recognizing this problem, the past decade has seen a dramatic upsurge of studies focused on zebrafish behavior (Kalueff et al., 2014). Social behavior of the zebrafish has started to be characterized too. The following section focuses on a specific form of zebrafish social behavior, shoaling. Shoaling may easily be induced and precisely quantified using juvenile and adult zebrafish in the laboratory, and more importantly, its analysis may have important translational relevance. 


\subsection{Human disorders with abnormal social behavior as a core symptom}

According to the Diagnostic and Statistical Manual of Mental Disorders $\left(5^{\text {th }}\right.$ ed.; DSM-5; American Psychiatric Association, 2013), abnormal social interaction is a core symptom for many different classes of disorders, but some argue that almost all human neurological and psychiatric disorders disrupt certain aspects of social function to a degree (Henry et al., 2016). Diseases that affect social interaction include ASD and the Fetal Alcohol Syndrome Disorders (FASD), which affect development of age appropriate communication and social skills (American Psychiatric Association, 2013).

Schizophrenia and other psychotic disorders, that alter cognitive processing, can also lead to social abnormalities, while mood and anxiety disorders such as depression or social anxiety disorders can limit social interaction (American Psychiatric Association, 2013). Personality disorders including sociopathy and psychopathy, disruptive and impulsive disorders (antisocial personality disorder and conduct disorder), and even substance abuse and addiction may lead to reduced affective control, increased social aggression and/or impaired social perception (American Psychiatric Association, 2013). Even such neurodegenerative disorders as age-related dementia, Parkinson's disease, and Alzheimer's disease may result in social behavior abnormalities related to emotional or motivational issues (American Psychiatric Association, 2013; Henry et al., 2016; Jones and Norton, 2015). Given the complexity of the above disorders and the importance of social behavior in our species, and given our limited understanding of the biology of this behavior, developing models of abnormal social interaction in social animals is an essential enterprise. The zebrafish has been suggested as a promising model for a variety 
of human CNS disorders and has been used across multiple disciplines as a tool to study human disorders with social abnormalities (Morris, 2009; Norton, 2013; Stewart et al., 2014; Tropepe and Sive, 2003). The complexity of zebrafish social behavior complements this species' practical advantages. It allows the induction and modeling of a potentially wide range of social abnormalities and a spectrum of severity of symptoms, a particularly useful feature of the zebrafish for modeling human disorders across the spectra of symptoms and severity. Implementing these models will require a better understanding of zebrafish social behavior and of the neural mechanisms that underlie it.

\subsection{Social behavior of zebrafish}

Characterization of social behavior of the zebrafish (Dahlbom et al., 2012; Miller and Gerlai, 2007) and specific features of social interaction (Qin et al., 2014; Saverino and Gerlai, 2008) have already begun. A typical feature of zebrafish social interaction is shoaling, the tendency to form and remain in a tight group (Miller and Gerlai, 2007). Shoaling is distinguished from schooling, the latter being associated with coordinated swimming direction, while the former simply represents the aggregation of individuals without synchronization of movement (Miller \& Gerlai, 2012). Both these forms of group forming behaviors have been observed, albeit under different circumstances, in adult zebrafish (Miller \& Gerlai, 2012). For example, shoaling tends to dominate in habituated fish, whereas schooling appears under aversive conditions or in response to a clear and present danger (Miller \& Gerlai, 2012). Although innate, shoaling is not observed in zebrafish younger than 7 days post-fertilization (dpf). But as the fish mature, shoaling appears (demonstrated by $15 \mathrm{dpf}$ ), and cohesion among shoal members significantly 
increases until the fish reach sexual maturity at age 3-4 months, after which shoaling plateaus (Buske and Gerlai, 2011; Dreosti et al, 2015; Hinz \& de Polaveija, 2017).

Shoaling may have negative consequences, for example, increased competition, aggressive encounters with shoal mates, and stress for subordinate fish due to social hierarchy (Gómez-Laplaza and Gerlai, 2011; Pavlidis et al., 2011). Nevertheless, adaptive advantages, including protection from predators, effective identification of food patches, and access to mates (Miller and Gerlai, 2007; Spence et al., 2008), appear to outweigh the disadvantages.

Besides shoaling, other social behavioral phenomena such as establishment of dominance hierarchy (Dahlbom et al., 2012; Larson et al., 2006), aggression (Teles and Oliveira, 2016; Way et al., 2015), courtship and reproductive behaviors have also been investigated in zebrafish (Coe et al., 2010; Ruhl et al., 2009). Similarly, there has also been a growing interest in characterizing non-social behaviors of zebrafish that may be related to social interaction and may allow better modeling of various human disorders (Seguin et al., 2016). Non-social behaviors that may influence social responses include locomotion, learning, anxiety, and fear responses (Al-Imari and Gerlai, 2008; Bencan et al., 2009; Lee et al., 2010; Maximino et al., 2010; Saili et al., 2012), but how these factors may influence social responses and how they may be regulated by social context remain unexplored.

While strain differences demonstrating the effects of genes on shoaling have been documented (Barba-Escobedo and Gould, 2012; Mahabir et al., 2013; Wright et al., 2003), no specific genes or quantitative trait loci associated with shoaling have been identified (Miller and Gerlai, 2007; 2011b). Similarly, while changes in the levels of 
neurotransmitters such as dopamine and serotonin have been found to be positively correlated with shoaling in zebrafish (Buske and Gerlai, 2011; Dahlbom et al., 2012; Saif et al., 2013; Shams et al., 2015), specific functional mechanisms and brain regions that control shoaling remain poorly understood. Nonetheless, reliable paradigms that allow manipulation and quantification of shoaling and related behaviors provide unique opportunities to predict, test, and understand biological mechanisms that regulate shoaling and, in turn, enhance social behavior research.

\subsection{How to induce and measure shoaling in zebrafish}

Shoaling is a robust social behavior typical of zebrafish that is readily observable in the wild (Spence et al., 2008; Suriyampola et al., 2016) and easily elicited in a laboratory setting by providing suitable social stimuli (Qin et al., 2014). For example, the existence or strength of shoaling may be tested using a choice task in which the test fish is placed singly in the middle of an aquarium flanked on opposite sides by social or control stimuli (Miller and Gerlai, 2007). The social stimulus can be a group of live conspecific fish, computer-animated images of zebrafish, or small robotic probes that physically emulate zebrafish (Abaid et al., 2012; Butail et al., 2014; Saverino and Gerlai, 2008), while the control may be an empty aquarium, a blank screen, or objects or images not resembling fish. The experimenter can make video recordings from the front of the tank (side-view) and analyze the recordings to quantify preference using various commercially available automated tracking programs or manual as well as computer aided event recorders. Tracking software applications can monitor movement of the test fish, record $x-y$ coordinates, and extract several swim path parameters, including time 
spent in proximity of the visual stimuli or distance from the stimulus side vs. the control side. These preference or choice tasks may also be utilized to investigate the effect of numerous aspects of the social stimulus, including shape, color, pattern, movements, location, number, density of the stimulus images.

On the other hand, more natural group dynamics can be investigated by placing multiple experimental fish (usually 4-10) together in an open-tank where they can freely move. In a freely moving shoal not only visual but also olfactory, auditory, and lateral line related information may be perceived by shoal members, and the behavior of the target fish may be influenced by the interaction between all members of the shoal (Engeszer et al., 2004). In this set up, the movements of the experimental fish are usually monitored from an overhang camera (bird's-eye view), which allows recording of numerous dynamic aspects of shoaling behavior (Buske and Gerlai, 2014). Behavioral parameters one may extract include the area occupied by the whole group, interindividual distances, nearest-neighbor distances, polarization of movement, excursions or fissures from the group (Buske and Gerlai, 2011; Miller and Gerlai, 2007; 2008; 2011a. 2011b; 2012; Miller et al., 2013; Shams et al., 2015). While lacking experimental control of the stimuli perceived (increased variability) and posing a greater challenge of the quantification of behavior (i.e., a larger number of dynamically moving agents to track and statistically analyze), the analysis of freely moving live shoals allows more precise description of the dynamic social responses of the zebrafish both in short (seconds) and longer (days, weeks or months) time scales (Miller and Gerlai, 2008; Buske and Gerlai, 2011). Both the monitoring of live shoals and the application of computerized social cues as animated images of conspecifics may be utilized to investigate changes of social 
behavior induced by a variety of experimental manipulations, including the delivery of different environmental stimuli (Engeszer et al., 2004; Gerlach et al., 2008; Moretz et al., 2007) or pharmacological agents (Coe et al., 2010; Buske and Gerlai, 2010).

\subsection{The effect of environmental stimuli on shoaling}

Interestingly, abiotic as well as biotic environmental factors can enhance or dampen social interaction. Abiotic factors such as substrate of home/testing tanks, speed of water flow, and presence of vegetation can affect social interaction, particularly aggressive and reproductive behaviors (Keck et al., 2015; Suriyampola et al., 2016). Presence of predators can influence shoal cohesion, while presence of social stimuli can affect anxiety and fear responses (Schaefer et al., 2015). The size and composition of the stimulus shoal are also important, and several features of shoal mates have been shown to also induce preferential responses in zebrafish, including sex and number of shoal members shown, sex ratio within the stimulus shoal, body size, stripe pattern, and pigmentation of stimulus fish (Kitevsky and Pyron, 2003; Ruhl and McRobert, 2005; Qin et al., 2014; Seguin \& Gerlai, 2017; Seguin et al., unpublished results).

Manipulation of the early social environment such as composition of the social rearing group and exposure to specific pigment or stripe pattern phenotypes can result in changes in subsequent social behavior and shoaling preference (Engeszer et al., 2004; Moretz et al., 2007). The absence of specific visual or odor cues from siblings for the first seven days of life has been shown to result in the reduction or loss of future kin recognition, while complete social isolation across development has been found to impair social behavior later in life (Engeszer et al., 2004; Gerlach et al., 2008). However, the 
effect of complete isolation has not been investigated past the larval stage, and thus we do not yet know whether effects may persist in adulthood.

In contrast, the effects of isolation of adult zebrafish have been relatively well studied. Isolation of adults prior to or during experimental manipulation is often employed as it allows the experimenter to individually recognize, monitoring, or manipulate the subjects across multiple occasions or trials (Al-Imari and Gerlai 2008; Teles et al., 2013). Although not specifically designed to analyze the effects of isolation per se, the isolation procedure may lead to numerous changes in social and non-social behaviors. Long social deprivation in adulthood (90 days) have been found to alter anxiety-related responses and to reduce whole-brain serotonin and whole-body cortisol levels in adult zebrafish (Shams et al., 2015). Behavioral paradigms, in particular social interaction tasks and tests of anxiety and locomotor activity, may be unreliable if prior social experience and social environment of the testing or stimulus fish are not taken into consideration. Underlying functional mechanisms such as specific brain areas and neurotransmitter systems that may be affected by acute, chronic, and developmental social isolation must be thoroughly investigated in the future.

\subsection{The effects of pharmacological agents on shoaling}

The zebrafish has been utilized for the screening and the analysis of the effects of several toxicants, teratogens, environmental pollutants and other pharmacologically active compounds. For example, exposure to pentylenetetrazol, valproic acid, or ethanol during early development has been shown to significantly affect subsequent development and expression of behavioral and physiological characteristics (Cowden et al., 2012; Lee 
et al., 2010; Zellner et al., 2011). Additionally, embryonic exposure to low doses of alcohol has been found to result in impaired shoaling, as well as abnormal functioning of the dopaminergic neurotransmitter system in adulthood (Buske and Gerlai, 2011; Fernandes and Gerlai, 2009; Fernandes et al., 2015b). Similarly, early transient exposure to synthetic estrogen (Ethinylestradiol) has been shown to affect adult breeding behavior and reproductive success (Coe et al., 2010).

The effects of anxiolytics, antidepressants and psychostimulants on the behavior of adult zebrafish have also been investigated (see Shams and Gerlai, 2016, for a review) but opioids and cannabinoids remain understudied (Gebauer et al., 2011). For instance, exposure to anxiolytics (fluoxetine, buspirone, or diazepam) leads to impaired shoaling (Giacomini et al., 2016). Shoaling may also be disrupted by lower doses of selective serotonin reuptake inhibitors (SSRIs) and other antidepressant drugs, but higher doses have been found to enhance social interaction in zebrafish (Schaefer et al., 2015). NMDA-receptor antagonism with MK801 has been found to induce social deficits, which can be reversed by certain antipsychotics (e.g. sulpiride) but not by others (e.g. haloperidol) (Savio et al., 2012). Acute nicotine treatment was found to reduce shoal cohesion, while chronic nicotine was found to slightly increase shoaling (Miller et al., 2013; Stewart et al., 2015b). Overall, these findings suggest that shoaling behavior is highly responsive to a large number of pharmacological agents as well as environmental manipulations. Although promising, these lines of research may be viewed only as pioneering with effects and mechanisms that are often not yet understood.

\subsection{Prospects for shoaling analysis to model human disorders}


Shoaling behavior in zebrafish can be a powerful tool that may permit modeling of social abnormalities found in various human disorders, even ones exhibiting varying degree of severity in symptoms and co-morbidities (Jones and Norton, 2015). Although not a comprehensive account of the literature on manipulation and measurement of shoaling in zebrafish, the reviewed studies hopefully demonstrate the utility of the zebrafish in general and the usefulness of measuring shoaling responses in particular. It should also be clear that we have significant gaps in our understanding of both the biological and the behavioral mechanisms of shoaling. Thus, the question remains open whether shoaling behavior meets the three criteria of a good animal model, namely face, construct and predictive validity. Nevertheless, given the powerful genetic and neurobiological tools developed for the zebrafish, and given the increasingly sophisticated methods custom designed for the analysis of its behavior, we hope that the above question will soon be answered in an affirmative manner.

One of the human diseases for which the zebrafish has been suggested as an excellent model (Tropepe \& Sive, 2003) and for which proper analysis of shoaling in zebrafish may be highly important is the ASD cluster, diseases characterized by abnormal social behavior. The following section reviews the use of zebrafish in modeling ASD.

\section{Behavioral, anatomical, and pharmacological phenotyping of zebrafish models of}

\section{ASD}

\subsection{What is ASD? Symptoms, prevalence and treatment options}

ASD are a group of neurodevelopmental conditions characterized by deficits in social interaction, poor communication, and repetitive behaviors or restricted interests 
(American Psychiatric Association, 2013). Although estimates vary, roughly 1\% of children worldwide are born with some form of ASD (Lai et al., 2014) and upwards of 1 in 68 children are diagnosed with ASD (Christensen et al., 2016). ASD are phenotypically heterogeneous, and patients can suffer not only from a wide range of severity in the core symptoms of social interaction and repetitive behaviors, but also may present with a variety of co-morbidities, including sensory sensitivity, motor function impairment, intellectual disability, gastrointestinal distress, sleep disruption, and epilepsy (Geschwind, 2009). ASD are highly heritable, and alterations in many genetic loci have been now been linked to ASD through analysis of copy number variants (CNVs), genome-wide association studies, and whole exome sequencing efforts (De Rubeis et al., 2014; Iossifov et al., 2014; Krishnan et al., 2016; Poelmans et al., 2013; Sanders et al., 2011).

The Simons Foundation Autism Research Initiative (SFARI) Gene Database, as of Sept 2016, lists 845 genes that have been linked to ASD, with roughly 65 genes and a handful of copy number variants (i.e. large stretches of duplicated or deleted DNA sequences that can even encompass many genes) considered to be high confidence ASD risk loci. Although network analyses of gene function and spatial-temporal co-expression have hinted at core ASD signaling pathways (Ellegood et al., 2015; Parikshak et al., 2013; Voineagu et al., 2011; Willsey et al., 2013), the genotypic and phenotypic diversity of ASD pose considerable challenges in identifying common underlying mechanisms, which in turn impede the search for effective treatments. Indeed, what is more notable in comparative analyses across 10-25 rodent ASD models is the observed phenotypic diversity rather than the areas of overlap (Ellegood et al., 2015; Ey et al, 2011). What is 
needed are good animal models for ASD in which these basic questions can be tackled. However, given the genetic diversity of ASD, the development of an appropriate set of animal models that can capture the phenotypic diversity of ASD is difficult, likely requiring a cross-comparative approach among a set of animals harboring mutations in the many ASD risk genes.

\subsection{Unique advantages of zebrafish in ASD modeling}

In light of the specific challenges involved in modelling complex, multi-genic disorders, especially the need to cost-effectively produce and phenotype dozens of genetic models that encompass the range of ASD phenotypes, the zebrafish model has several attractive features.. In particular, zebrafish at their larval stages are optically transparent and develop externally, allowing for the direct visualization of morphological changes during early development (Tropepe \& Sive, 2003). Brain development is rapid, as the larval zebrafish goes from a single fertilized cell to an embryo with fully established body axes and an embryonic brain with distinct vertebrate morphology and anatomical subdivisions by 28 hours post fertilization (hpf) (Kimmel et al., 1995). Within $5 \mathrm{dpf}$, major neurotransmitter and peptidergic neuronal subtypes are in place and the larvae are capable of complex behaviors (Panula et al., 2010). The larval brain is small enough to also allow whole mount antibody staining and imaging for a global visualization of the formation of major axon tracts, proper patterning of neuropil, and the development of major neurotransmitter classes (Turner et al., 2014; Wilson et al., 1990). The differentiation, migration, and morphological characteristics of many cells can be readily tracked in vivo with an increasing collection of zebrafish driver lines that express 
fluorescent proteins (e.g. green fluorescent protein, GFP) in cells of interest (Kawakami et al., 2016). Many transgenic lines are available that label neurons with particular relevance to ASD, such as inhibitory GABAergic interneurons (Zerucha et al., 2000) or neurons that express the neuropeptide, oxytocin (Blechman et al., 2011), which is thought to regulate social interaction in many species (Peñagarikano, 2016). Time lapse movies of intact animals over several days can visualize the differentiation and migration of neurons (Satou et al., 2013), the projection of axons and dendrites and the formation and destruction of synapses (Appelbaum et al., 2010; Shamay-Ramot et al., 2015), all processes whose dysfunction have been implicated in ASD. Furthermore, transgenic driver lines can be used to manipulate neurons for interrogation of neuronal function, for example, to visualize neuronal activity using genetically encoded calcium indicators (Ahrens et al., 2012), to ablate neurons with cell-specific toxins or lasers (Mathias et al., 2014; Roeser and Baier, 2003), or to activate or inhibit neurons with small molecules (i.e. chemogenetics) or with light (i.e. optogenetics) (Chen et al., 2016; Portugues et al., 2013). "Snapshots" of whole brain activity can also be taken using in situ hybridization of the immediate early gene $c$-fos, a marker of neuronal activity (Ellis et al., 2012), or with antibody staining for another activity marker, phosphorylated ERK (Randlett et al., 2015). Together, this collection of tools for the interrogation of neural development and function will enable in vivo dissection of the changes that occur when ASD risk genes are mutated.

Another attractive feature of the zebrafish model is the rich repertoire of behaviors that appear at early developmental ages. At $24 \mathrm{hpf}$, larvae are already capable of simple behaviors, such as coiling in response to touch (Saint-Amant and Drapeau, 
1998). By 3 dpf, the retina is wired to respond to visual stimuli (Fadool and Dowling, 2008), and the larvae can spontaneously swim. By 5 dpf, the larvae are free swimming and exhibit complex behaviors that integrate internal and external stimuli across multiple sensory modalities, such as hunting (Budick and O’Malley, 2000), motor learning (Kawashima et al., 2016), and sleep (Zhdanova et al., 2001). Many of the major behavioral co-morbidities with relevance to ASD, including sleep-wake disturbances, seizures, and gastrointestinal problems (Geschwind, 2009), can be studied in these early larval stages. Using a high speed camera, detailed kinematic analysis of larval spontaneous and visually-evoked motor responses can detect alterations in muscle coordination, inter-bout intervals, swim speed, and other parameters (Fontaine et al., 2008; Severi et al., 2014). Sensory sensitivity can be measured by examining sensorimotor gating and habituation of startle responses to acoustic (Burgess and Granato, 2007; Wolman et al., 2011) or visual (Emran et al., 2007) stimuli. Long term behavioral tracking over several days can identify changes in sleep bout patterns across the 24-hour day-night cycle (Rihel et al., 2010). Spontaneous and drug-induced seizures (e.g. by exposing larvae to the GABA-receptor antagonist, Pentylenetetrazol, PTZ) can be observed using both behavioral and electrophysiological criteria (Baraban et al., 2005). Non-behavioral perturbations can also be investigated; for example, gut function can be elegantly tracked in zebrafish by feeding the larvae fluorescent material and measuring transit time (Field et al., 2009). Importantly, for most of these behavioral assays, dozens or hundreds, even thousands of larvae can be simultaneously measured, which facilities drug screening via direct application of small molecules into the larval home-tank water (Rihel and Schier, 2012). 
Zebrafish are also highly social animals, showing a clear preference for conspecifics in a simple T-maze by three weeks of age (Dreosti et al., 2015). The first significant signs of shoaling (group forming) have been demonstrated by the age of 15 dpf (Buske \& Gerlai, 2011; Dreosti et al, 2015; Hinz \& de Polaveija, 2017), and sophisticated and complex social interactions have been already described (see section above). Although obviously of great interest for the study of ASD, it is not yet clear to what extent the circuits, and the neurobiological mechanisms in general, that control social behavior in zebrafish are conserved across fish and mammals, including humans. Nevertheless, linking social interaction to discrete circuits that are perturbed by mutations in ASD risk genes would be a promising future avenue of research.

\subsection{Genetics of ASD in zebrafish}

Most human ASD-risk genes have been found conserved in the zebrafish. From genome searches, we estimate $>90 \%$ of human ASD genes have a clear ortholog in zebrafish (Rihel, personal observation; also see Tropepe \& Sive, 2003; McCammon \& Sive, 2015; Wright \& Washbourne, 2011). To date, most of the zebrafish studies of ASD-associated genes have employed a knockdown approach using morpholinos (MOs), which are anti-sense oligonucleotide-like molecules with a specialized backbone that can block either translation or splicing of targeted transcripts-of-interest when injected into the zebrafish embryo (Nasevicius and Ekker, 2000). Typically, these studies have focused on alterations in early neuronal development following MO injection, with a few studies also examining effects on sensory-evoked locomotor behavior and seizures. For example, MO knockdown of the ASD gene met (a tyrosine kinase receptor) led to reduced cell 
proliferation in the cerebellum (Elsen et al., 2009), knockdowns of either zebrafish shankla, shanklb, or syngaplb delayed brain development and caused seizures and altered motility (Gauthier et al., 2010; Kozol et al., 2015), and MOs targeting autism susceptibility candidate 2 (auts2) led to reduced head size and loss of neurons (Oksenberg et al., 2013). Other zebrafish knockdown studies focused particularly on head size, which is often altered in ASD patients, including targeted knockdowns of kcdt13 (Golzio et al., 2012) and chd8 (Bernier et al., 2014; Sugathan et al., 2014), which caused macrocephaly. In light of concerns that MO injections cause unwanted and poorly understood side effects, which often include alterations to head size, neuronal development, and perturbations of motor neuron function, most zebrafish studies of ASD using MOs should now be interpreted with great caution (Lawson, 2016). Systematic comparison of mutant and morphant data demonstrated that MOs often fail to recapitulate genetic loss of function mutant phenotypes, suggesting that MOs are causing non-specific phenotypes (Kok et al., 2015, although see Rossi et al., 2015 for other specific possibilities). When evaluating ASD morphant phenotypes, it is helpful to keep in mind the published criteria for analysis of MOs (Eisen and Smith, 2008), including 1) phenotypic rescue by co-injecting mRNA, 2) using two independent MOs targeting the same gene, and 3) confirmation by comparison to a mutant in the same gene. Perhaps the tendency of ASD-risk genes to be unusually large in both humans and zebrafish (King et al., 2013; Kozol et al., 2016) has precluded easy demonstration of morphant rescue with functional mRNA, but this only emphasizes the need for confirmation of morphant ASD phenotypes with genetic mutations. 
Going forward, the availability and ease of modern gene editing techniques will become the gold standard for the analysis of ASD gene function in zebrafish. However, editing ASD genes in zebrafish poses its own challenges. Many of the large ASD genes undergo complex alternative splicing, so mutants must be carefully evaluated for true loss of function. Intriguingly, some 50-60\% of ASD genes are duplicated in zebrafish (compared to roughly $20 \%$ across the mammalian genome), which may require the targeting of each copy to create a loss of function ASD gene model (Kozol et al., 2016). Nevertheless, often the expression of these duplications are restricted to non-overlapping tissues or take on separated functions, thereby affording the opportunity to dissect genes with pleiotropic functions (Conant and Wolfe, 2008). In any case, the advent of the powerful and straightforward Crispr/Cas9 gene editing technology, which can be multiplexed to simultaneously generate mutant alleles in the same animal, makes functional gene redundancy a more easily tackled problem in zebrafish (Jao et al., 2013). The few zebrafish mutants in ASD risk genes generated to date point to alterations in excitatory-inhibitory balance. For example, null mutants for the Rett Syndrome gene, mecp2, have defects in spontaneous swimming bouts (Pietri et al., 2013), while mutants that lack both zebrafish orthologs of the ASD risk gene contactin associated protein-like 2 (cntnap2) have a large reduction in GABAergic interneurons, seizure susceptibility, and behavioral hyperactivity at night (Hoffman et al., 2016). These conclusions are broadly consistent with rodent models harboring mutations in MeCP2 and CNTNAP2. For example, several MeCP2 mutant lines have been generated that have motor defects and lowered cortical activity due to alterations in excitatory-inhibitory balance (e.g. Dani et al., 2005). As in the zebrafish, CNTNAP2 mouse mutants have epilepsy, a loss of 
migrating GABAergic interneurons, and hyperlocomotor activity in an open field test (Penagarikano et al., 2011). While these zebrafish ASD mutant examples are consistent with phenotypes in both humans and rodent models, these mutant phenotypes are generally much milder than observed in morphant studies, further underscoring the importance of testing gene function with mutational analysis. How well zebrafish models of other ASD risk genes will concord with rodent studies remains an open question until the relevant mutants are generated and tested. Importantly, the extent to which zebrafish ASD mutants will exhibit alterations in social and repetitive behaviors remains unknown.

\subsection{Integration of imaging, behavior, genetics, circuits, and drugs}

The power of the larval zebrafish model will rely on the ability to rapidly identify how genetic lesions in ASD risk genes alter the development of neuronal circuits that modulate ASD-associated behaviors and then to leverage small molecule screens to find drugs that can revert mutant phenotypes. An example of such an approach is the recent study of the zebrafish cntnap2 mutants by Hoffman et al. (2016). By comparing the nighttime hyperactive phenotype of cntnap 2 mutants to behaviors induced in wild larvae exposed to a large panel of small molecules, they were able not only to predict NMDA receptor antagonists as potent sensitizers of the mutant behavior, but they also discovered that estrogenic compounds, including the phytoestrogen biochaninA, selectively rescued the phenotype. This kind of "predictive pharmacology" offers the potential to avoid labor intensive drug screening on mutant backgrounds by first preselecting promising candidate compounds for a more targeted search. 
The analysis of cntnap2 mutants also established a core set of ASD related phenotypes that span neuronal development (i.e. whole brain imaging of GABAergic interneurons and other cell types), seizure propensity, sleep, and drug sensitivities (Hoffman et al., 2016). As more zebrafish mutants are generated for the many other ASD genes, it will be of great interest to determine the degree of overlap and divergence observed among these phenotypes across the various genetic lesions. How much of the variation among ASD mutants will converge on the same brain structures, the same circuits, and the same drugs? Another major open question is the extent to which phenotypic diversity among zebrafish ASD models will predictively overlap with the diversity of phenotypes in human patients. Might the animal models suggest phenotypic groupings among risk genes that can help to further subdivide the spectrum of autistic patients into distinct classes? The answer to this question may have important implications on the search for treatments as well as on clinical practice, as therapies may need to be tailored to the distinctive biological features of each patient subset. Thus, the generation and phenotyping of dozens of zebrafish models of ASD will offer insights not only into core aspects shared across all the models but will also be a powerful tool for the analysis of the pleiotropic features of ASD biology.

Tailoring therapies to particular forms of ASD will require identification of drugs that act through molecular mechanisms potentially unique to the ASD subset targeted. Drug screening is a labor intensive and time consuming process. A possible short cut that may save time and effort is to focus on small molecules already "prescreened" by nature. The following section discusses this approach with a focus on anxiety and epilepsy related efficacy. 


\section{Screening of natural products using zebrafish behavior: epilepsy and anxiety}

\subsection{Drug discovery and Zebrafish}

Conventional drug discovery schemes focus on: (a) target discovery and validation; (b) screening hits-to-leads libraries; and (c) lead optimization of pharmacological and toxicological properties. In addition, the extremely high failure rate of many preclinical drug candidates is attributed in part to their undesirable pharmacological or toxicological properties. Zebrafish are well-suited for high throughput screening for small molecules and genetic mutations generated, for example, by the increasingly powerful Crispr/Cas9 system. Zebrafish-based high throughput screening has been suggested by many authors, but remains underutilized for CNS drugs despite that phenotypic screening using these fish may aid drug discovery. As previously mentioned, the ease of maintenance and breeding of zebrafish, and their similarities to rodents render these fish amenable for behavioral screens. Moreover, many of the behavioral tests used in rats are easily adapted to zebrafish (Kalueff et al., 2014), making them ideal to study human disorders. For example, zebrafish mutants have been identified even for rare human disorders, including Dravet syndrome (heritable form of pediatric epilepsy), and they have been utilized in screening for approved drugs to find possible treatments for such disorders (Baraban el al, 2013, Dinday and Baraban, 2015).

\subsection{Natural products}

The use of drugs obtained from nature is not new. At least $25 \%$ of the drugs employed currently in the human clinic have been derived from natural products 
(Newman and Cragg 2012, Cragg and Newman 2013). However, screening natural products for specific biological properties is a daunting task, as one often has to start with a complex mixture of unknown compounds, a problem that may be resolved with purification. The combination of purification and zebrafish-based screening has already been successfully employed (Bohni et al., 2013; Brillatz et al. 2016; Challal et al., 2014; Crawford et al., 2014; Tabassum et al., 2015).

A significant number of people consume natural supplements without validation of the effectiveness of the active ingredients of these supplements and without a concern for their safety or possible interactions with conventional (e.g. FDA approved) drugs. Thus, it is important to characterize such natural supplements and identify their active ingredients, which later could be tested under controlled conditions in preclinical as well as human trials. The need for such efforts is clear, given that conventional drugs have often been found ineffective, or difficult to develop. There are pharmacological treatments for epilepsy and for anxiety, however, the efficacy of pharmacotherapy is suboptimal. Approximately $30 \%$ of patients do not respond well to conventional anticonvulsants or develop resistance to such drugs (Lazarowski et al., 2015). In addition, epileptic patients have a higher incidence of comorbidity of depression and anxiety (Tellez-Zenteno et al., 2007), further highlighting the need to develop safer therapeutic compounds that may be able to target the complex interactions of concurrent conditions.

Ethnopharmacology-based screening may result in faster and cheaper identification and optimization of active ingredients. In this section, a few examples will focus on discoveries in one author's lab (Ortiz), who has used zebrafish to test extracts from Valeriana officinalis and Citrus aurantium for potential anticonvulsant and anxiolytic 
activity and have utilized the zebrafish to identify possible mechanisms underlying the effect of these extracts.

\subsection{Anxiety screening}

Two behavioral paradigms sensitive to detect anxiety-like responses in the zebrafish, the open field and the light-dark preference test, have been used to investigate the effects of natural products and other small molecules. These behavioral tests are direct adaptations from tests originally developed for laboratory rodents. In the light-dark preference task the anti-epileptic and anxiolytic compound, clonazepam, has been found to increase the time spent in the light side, while caffeine, a stimulant, markedly reduced this response (del Valle-Mojica and Ortiz, 2012). The fish were tested in groups (typically 3 fish at a time) because their behavior is less variable in such setting (del Valle-Mojica and Ortiz, 2012) (also se figure 1).

\section{Valeriana officinalis}

Valerian (Valeriana officinalis) is a perennial flowering plant native to Europe and parts of Asia, and also introduced to North America, and is used as an herbal supplement. Different formulations of valerian roots are sold to reduce anxiety (anxiolytic) or as a sleep aid [http://ods.od.nih.gov/pdf/factsheets/Valerian-HealthProfessional.pdf]. The Ortiz laboratory has found that aqueous valerian extract and valerenic acid (a marker compound of $V$. officinalis) both increased the time zebrafish spend in the light side in the light dark preference task, similarly to clonazepam (del Valle-Mojica and Ortiz, 2012). Furthermore, studies have identified interactions of valerian extracts with the 
metabotropic glutamate receptors, mGluR I and mGluR II receptors in vitro (Del ValleMojica et al., 2011) and in adult zebrafish (del Valle-Mojica and Ortiz, 2012).

\section{Citrus aurantium}

Sour orange (Citrus aurantium) is a tree native to southeast Asia, but it has been spread by humans to many other parts of the world. The peel of the Citrus aurantium's fruit is often used as weight loss supplement, as a replacement for ephedra (Haaz et al., 2010). Notably, Citrus aurantium leaf extracts are also used to treat anxiety in Puerto Rican folklore medicine and in other cultures (Alvarado-Guzmán, 2009, Hernandez et al., 1984; Pimenta et al., 2016). Aqueous extracts devoid of the sympathomimetic compounds present in the fruits' peel (Rosa-Falero et al., 2015), exert anxiolytic effects in zebrafish in both the open field and the light-dark preference tasks (Ortiz, unpublished results, also see figure 2). In vitro, Citrus aurantium interacts with all GluRs (RosaFalero et al., 2015). Citrus aurantium extracts also display a "U" shaped dose response that has been difficult to analyze (Ortiz, unpublished results).

\subsection{Anticonvulsant screening}

Baraban et al. (2005) pioneered the use of pentylenetetrazol (PTZ) to induce seizures in the zebrafish. This method has been adapted to screen extracts with potential anticonvulsant properties (Torres-Hernandez et al., 2015, Rosa-Falero et al., 2015).

\section{Valeriana officinalis}


Valeriana officinalis and valerenic acid have anticonvulsant properties in adult zebrafish (Torres-Hernandez et al., 2015). Moreover, valerian extracts potentiate the anticonvulsant effects of clonazepam and of phenytoin. Adenosine (A1) receptors and group I and II metabotropic glutamate receptors (mGluRs) interact with the effect of valerian, and alter the onset of PTZ-induced convulsions (Torres-Hernandesz et al., 2015; 2016). For example, antagonist for adenosine A2 receptors and group III mGluRs have been found to significantly reduce the effect of valerian (Torres-Hernandesz et al., 2015; 2016). Last, the effects of valerian extract were reduced by pre-treatment with selective antagonists for $\mathrm{GABA}_{\mathrm{A}}$, AMPA and KA (ionotropic glutamate receptors), and for group II mGluRs receptors. (Torres-Hernandez et al., submitted).

In larval zebrafish, Valeriana officinalis and valerenic acid have been found to reverse PTZ-induced alterations in swimming behavior (Torres-Hernandez et al., 2016). Moreover, the effects of PTZ on the immediate early genes $c$-fos, bdnf, and npas 4 genes have been found to be reversed by Valeriana officinalis and valerenic acid (TorresHernandez et al., 2016).

\section{Citrus aurantium}

Citrus aurantium's extracts are used in Puerto Rico and other cultures in folklore medicine to treat anxiety (Alvarado-Guzmán 2009; Hernandez et al., 1984; Pimenta et al., 2016). An aqueous extract made from $C$. aurantium's leaves has anticonvulsant properties in a PTZ-induced seizure model of zebrafish (Rosa-Falero et al., 2014). Preliminary results (Ortiz unpublished) suggest that NMDA- receptors and mGluRs I and II may mediate the anticonvulsant effects of Citrus aurantium. Moreover, Citrus 
aurantium extracts potentiate the anticonvulsant effects of phenytoin and of valproic acid (Rosa-Falero et al. unpublished results). Activity-guided screening using zebrafish of different fractions (subsets of small molecules fractionated based upon biochemical properties) is currently being pursued in an attempt to refine the molecules involved.

\subsection{Future Prospects for Natural Product Screening in Zebrafish}

The above discussed studies demonstrate that $V$. officinalis and C. aurantium extracts have anticonvulsant and anxiolytic properties. In addition, although the specific active ingredients of the extracts are awaiting confirmation, some mechanisms mediating these effects, notably mGluRs, have already been indicated. It appears that the zebrafish will be a useful laboratory animal for the identification of psychoactive compounds from natural extracts. First, zebrafish behavioral phenotypical screening may allow one to identify biological activity of complex extracts without having to know in advance what brain areas, brain functions, and what molecular mechanisms are affected. Once such activity is found, identification of the active ingredients or the combination of active ingredients may require thorough and time consuming fractionation and chemical composition analyses, which again may be conducted more cheaply and efficiently with the zebrafish.

Anxiety and epilepsy are but two of many central nervous system disorders one may be able to model using the zebrafish. Modeling most human disorders, however, is often problematic because we often do not know the cause of the disease. This is not an issue for the disorder cluster, the Fetal Alcohol Spectrum Disorders (FASD), discussed in 
the subsequent section. These diseases are clearly caused by alcohol consumed during pregnancy.

\section{Developing a zebrafish model of milder forms of FASD}

Modeling human diseases with the use of laboratory organisms has several advantages compared to studying the disease directly in humans. These include better laboratory control of known factors that affect the disease under study, better experimental techniques available for manipulation of the biological mechanisms suspected to underlie the disease, and also importantly reduced cost and increased efficiency of research. As the previous sections have already demonstrated, all of these advantages characterize research with the zebrafish. Using animals to study alcohol related human disorders has yet another advantage compared to models of many other disorders: the cause of the disease (alcohol exposure) is known.

\subsection{What is FASD? Symptoms, prevalence and treatment options}

FASD encompass a cluster of diseases with differing severity and varied symptomatology (Nash et al., 2006; 2008; Streissguth et al., 1978). The common underlying feature of these diseases is their cause: alcohol consumption during pregnancy. Although these diseases could be completely eradicated by not drinking alcohol, fetal alcohol exposure remains the single most frequent cause of intellectual disability in children (Mattson et al., 2011; Stratton et al., 1996). The prevalence of FASD is estimated to range from 1 to in 5\% in the Western world (Sampson et al., 1997; May et al., 2009), an alarmingly large number given the known deleterious effects of alcohol on the fetus. The severity or type of symptoms within FASD define different 
subsets or categories of the disorder (Stratton et al., 1996). The most severe form of the disease is Fetal Alcohol Syndrome (FAS), which is often characterized by clearly observable anatomical, for example, facial abnormalities, growth retardation and significant impairment in executive function, learning and memory, language, visualspatial ability, motor function, and attention (Stratton et al., 1996; Mattson et al., 2011). Milder forms of the disease have different diagnostic names, for example, alcohol related neurological disorder (or ARND), but common to all of them is the lack of gross anatomical changes (Stratton et al., 1996). These disorders may be milder, but patients suffering from them do exhibit numerous behavioral impairments, including cognitive impairment. Abnormal social behavior is more recently recognized as one of the hallmarks of the milder forms of FASD (Stevens et al., 2015). These abnormalities may manifest as reduced empathy and perspective taking, decreased ability to solve social problems, and difficulty interpreting mental states and emotions of others (Greenbaum et al., 2009; Stevens et al., 2015). Despite the high prevalence of FASD and the life-long adversity faced by patients suffering from this disease, no treatment is available, and even clear diagnostic criteria, such as testable biomarkers, are absent. To address these serious issues, numerous animal models have been proposed, zebrafish being one of the newest, but perhaps most powerful, among them (Gerlai, 2015; Patten et al., 2014).

\subsection{Zebrafish models of FASD}

In addition to the advantages of the zebrafish as a biomedical research organism discussed in the introduction, it is also an ideal model organism for the analysis of alcohol effects in general, and for the analysis of the effects of alcohol on the developing 
embryo in particular. The eggs of zebrafish are externally fertilized, develop outside the mother and without her influence. This was a major advantage for developmental biologists who could view the development of the embryo under the microscope without having to employ invasive methods. This is also a major advantage from the perspective of neuroscientists interested in modeling FASD. The eggs and the embryos inside may be exposed to alcohol at any specific desired stage of development for precisely preset periods of time and at specific experimentally determined doses (Fernandes and Gerlai, 2009). This level of experimental control cannot be achieved in mammalian systems. This feature may be viewed as a disadvantage, as it does not realistically mimic what happens in case of mammalian embryos, which develop in the uterus and are protected by numerous defense mechanisms of their mother. However, the fish model may also be viewed as having advantages over mammalian (e.g. rat or mouse) models exactly because it is devoid of the complications associated with maternal physiology and maternal care (Fernandes and Gerlai, 2009). It is a simplistic and reductionist model that seeks to address the questions of how alcohol affects the development of the embryonic brain and how this alteration may manifest in behavior later in life.

\subsection{A realistic zebrafish model of the most prevalent, milder, forms of the disease}

Recognizing the above advantages of zebrafish, this species was first utilized in modeling FAS, the most severe forms of FASD. Zebrafish embryos exposed to high doses of alcohol (1.5-2.9\% vol/vol alcohol) for prolonged periods of time (from several hours to days) indeed developed robust anatomical abnormalities including microphthalmia, heart defects, enlarged body cavities, increased mortality (Arenzana et 
al., 2006; Bilotta et al., 2004; Carvan III et al., 2004). These early zebrafish FASD models were highly useful as they provided clear experimental evidence that alcohol affects the developing embryo even in fish, and the resulting anatomical abnormalities mimic many of the features seen in the severe forms of the disease in humans.

However, these early zebrafish models did not mimic what happens in the most prevalent forms of the disease, the milder cases of FASD. The milder forms of FASD are likely the result of exposure to lower level and less frequent consumption of alcohol. Fernandes and Gerlai (2009) tried to mimic this alcohol exposure by administering small doses (up to $1 \%$, vol/vol \% bath concentration) of alcohol for only a short period (2 hours) to eggs of zebrafish at their $24^{\text {th }}$ hpf stage. This study importantly also analyzed how much alcohol actually reached the embryo inside the egg (the chorion, the eggshell, is protective), and found that depending on dose, about $1 / 25^{\text {th }}$ to $1 / 30^{\text {th }}$ of the external bath concentration of alcohol could be detected inside the egg. That is, in case of $1 \%$ external bath concentration, $0.04 \%$ alcohol reached the embryo (Fernandes and Gerlai, 2009). Considering the North American legal limit of blood alcohol concentration for driving being $0.08 \%$, the above value is within a "physiological", range, at least as compared to typical human exposures.

\subsection{Behavioral effects of exposure to low concentration of alcohol during embryonic}

\section{development in zebrafish}

Zebrafish exposed to alcohol at 24 hpf for 2 hours at external bath concentrations up to $1 \%$ (vol/vol) survived the treatment without any obvious signs of abnormality. These fish grew with the same rate as their control counterparts, appeared to move normally, 
and their physical appearance was also of a typical wild type healthy zebrafish. Detailed analysis of their motor function also did not reveal any defects (Fernandes and Gerlai, 2009). However, when tested for their responses to social stimuli (images of moving conspecifics, described earlier), the alcohol exposed fish showed a significant impairment. Upon the presentation of these images, normal control fish approached the images and stayed in close proximity of the images. However, alcohol exposed fish stayed significantly farther from the images (Fernandes and Gerlai, 2009). It is important to emphasize that the impairment was found in adult, about 6 months old, fully developed, sexually mature zebrafish. In fact, subsequent studies demonstrated that the impairment persisted in much older (2 years old) zebrafish, as well (Fernandes et al., 2015a). Importantly, this behavioral impairment was found highly dose dependent (figure $3 \mathrm{~A})$. At the lowest employed dose $(0.25 \%$ alcohol, vol/vol $)$ the impairment was significant but only modest, but at the highest dose (1.00\% alcohol, vol/vol), the impairment was severe. In fact, fish exposed to the highest alcohol concentration during their embryonic development did not respond to the conspecific images at all when tested at their adult stage; that is, the distance they maintained from the image presentation screen during image presentation was statistically indistinguishable from the distance they kept from the screen before the presentation begin. Bathing the eggs in $1 \%$ alcohol for 2 hours at $24 \mathrm{hpf}$ abolished the response to conspecific images in the adult fish (Fernandes and Gerlai, 2009).

What could be behind this impairment? There may be many reasons the alcohol exposed fish did not properly shoal. One possibility is that they were unable to perform the appropriate motor responses due to impaired motor function. This working hypothesis 
turned out to be incorrect as analysis of the movement patterns of the embryonic alcohol exposed fish revealed no abnormalities (Fernandes and Gerlai, 2009). The general activity of the alcohol exposed fish, as measured by distance swum, was statistically indistinguishable from that of control fish. Similarly, the top speed, the number of fast and slow swim episodes, the number and degree and direction of turning performed by the alcohol exposed fish were statistically indistinguishable from the behavior of control fish. Another possible reason why the alcohol exposed fish showed impaired shoaling could be compromised vision, possibly from alcohol exposure during ontogenesis disrupting the development of the eye or other parts of the visual system. However, this working hypothesis was also found unlikely. The direct evidence against it came from the study performed by Fernandes and Gerlai (2009), who found that moving images of conspecifics elicited two distinct behavioral responses in healthy normal zebrafish: one, the classic shoaling response (staying close to the images); and two, reduction of swimming activity, i.e. slowing down. Interestingly, the latter response remained unaffected in embryonic alcohol exposed fish (Fernandes and Gerlai, 2009). That is, although these fish suffered from a significant impairment in their shoaling response, they reduced their swim speed and general activity upon presentation of the animated conspecific images just like control fish did. The latter response is only possible if the alcohol exposed fish could see the images. The indirect evidence for vision not explaining the reduced shoaling response came from a study that investigated freely moving shoals of zebrafish. Buske and Gerlai (2011) exposed zebrafish to alcohol during their embryonic development in the same manner as was employed in the Fernandes and Gerlai (2009) study but investigated the effect of the manipulation in freely moving 
zebrafish tested in a large open tank. In this set up too, embryonic alcohol exposed zebrafish were found to exhibit impaired shoaling as measured by a farther swimming distance between each other compared to control fish. Given that blind cave tetras as well as experimentally blinded shoaling fish of other species have been demonstrated to shoal normally (Gregson and Burt de Perera, 2007; Partridge and Pitcher, 1980), the role of visual impairment underlying the observed embryonic alcohol induced changes in shoaling seems unlikely (Buske and Gerlai, 2011). The third possibility that may explain the observed embryonic alcohol exposure induced impairment of shoaling responses we considered is altered fear and/or anxiety. One of the main adaptive functions of shoaling is believed to be predator avoidance, as more fish in a group may be able to detect an approaching predator sooner (Pitcher, 1993). Additionally, selecting a single target among many moving targets may be more difficult for the predator (Krause and Ruxton, 2002). Irrespective of what may be the actual function of shoaling, however, it has been shown to increase under aversive conditions. For example, the inter-individual distance among shoal members decrease in novel environments (mildly aversive context) and increase once the fish are habituated (Miller and Gerlai, 2007; Speedie and Gerlai, 2008). A fast-approaching predator triggers a rapid escape response (dispersal of the shoal) followed by a tightening of shoal cohesion (reduction of inter-individual distance among shoal members). Thus, it is possible that the impaired shoaling response observed in embryonic alcohol exposed zebrafish was the result of altered (reduced or enhanced) fear and/or anxiety in these fish. However, this working hypothesis also turned out to be incorrect. Seguin et al., (2016) found that zebrafish exposed to alcohol in the same manner, at the same developmental stage and with the same concentrations and exposure 
period as conducted by Fernandes and Gerlai (2009), exhibited no alterations under aversive conditions or in response to the appearance of a moving image of a sympatric fish predator. What could explain the abnormal responses to social stimuli then?

There are two remaining possibilities to consider. First, zebrafish exposed to alcohol during embryonic development may not recognize their conspecifics as such, and thus do not want to shoal with them. This hypothesis remains untested, although it appears unlikely to be correct. In nature, zebrafish form mix species shoals, mingling with fish of others species that have similar body size and general shape (Engeszer et al., 2007). In the laboratory too, these fish show shoaling responses towards animated fish images whose features differ from those of conspecifics (Saverino and Gerlai, 2008). The second possibility is that for some reason, although the embryonic alcohol treated zebrafish do recognize the images (or their conspecific shoal members) as appropriate shoal mates, their motivation to join the shoal is diminished. The dopaminergic system is known to be involved in reward. Could the dopaminergic system be impaired in embryonic alcohol exposed zebrafish?

\subsection{Potential mechanisms underlying embryonic alcohol exposure induced}

\section{behavioral abnormalities in zebrafish}

Alcohol is known to interact with a large number of molecular targets and in a concentration and exposure regimen dependent manner. Exposure to alcohol during embryonic development may lead to an even more complex effect profile, given that the initial alcohol induced changes may be followed by an avalanche of subsequently modified secondary responses. Given the expected complexity of such changes, one may 
need to conduct a comprehensive analysis of gene expression changes using deepsequencing technologies or more traditional DNA microarrays, a drug screen to test for compounds that may alter the embryonic alcohol induced changes, or a genetic screen to identify mutated genes that may exacerbate, diminish or negate the embryonic alcohol induced changes, to mention but a few approaches one could take. However, such comprehensive analyses are expensive and time consuming. Instead, as a first step, a hypothesis driven proof of concept analysis was conducted to test whether the dopaminergic system of the embryonic alcohol exposed fish have been altered. The rationale for this choice was supported by multiple findings. First, the sight of conspecifics was found to be rewarding; specifically, the presentation of live stimulus fish was demonstrated to serve as an unconditioned stimulus (reinforcement) in a classical conditioning paradigm (Al-Imari and Gerlai, 2008). Second, using a dopamine D1-receptor antagonist Scerbina et al. (2012) have been able to impair (reduce or abolish) shoaling, an effect that was concentration dependent. Third, and perhaps most importantly, Saif et al. (2013) found that the appearance of conspecific images to induce an increase of the levels of dopamine and DOPAC (dopamine's metabolite) in the zebrafish brain.

For the above reasons, Gerlai and his colleagues decided to measure levels of dopamine and DOPAC in embryonic alcohol exposed fish. Using HPLC (high-precision liquid chromatography) specifically developed for the detection of neurochemicals in zebrafish (Chatterjee and Gerlai, 2009), the amount of dopamine and DOPAC was analyzed from whole brain extracts of zebrafish. Zebrafish from age 15 to $102 \mathrm{dpf}$ were tested, and the relative amount of dopamine and DOPAC was found to significantly 
increase as the fish developed (Mahabir et al., 2013). Importantly, however, this agedependent maturation of the dopaminergic system was significantly blunted or arrested in fish exposed to alcohol during their embryonic development. In other words, a significant linear alcohol concentration dependent effect was demonstrated, with lower doses having a milder (yet still significant) and the higher doses more robust effect (Mahabir et al., 2013). Interestingly, further analysis revealed that basal levels of dopamine or DOPAC were unaltered by embryonic alcohol treatment. Fish that were housed alone and kept unstimulated by social cues exhibited similar levels of dopamine and DOPAC irrespective of whether they were alcohol treated or control (Fernandes et al., 2015b). However, and importantly, upon delivery of a social stimulus, control fish responded with a robust and significant increase of dopamine and DOPAC levels, while those fish that were exposed to alcohol during their embryonic development did not (Fernandes et al., 2015b) (figure 3B). These results thus confirm that embryonic alcohol exposure disrupts the ability of zebrafish to properly respond to social stimuli both at the level of dopaminergic neurochemistry and also at the level of behavior (shoaling). The finding of unaltered basal dopamine and DOPAC levels imply that dopamine production and release were unaltered in the embryonic alcohol exposed fish. Thus, one could speculate that the number of dopaminergic neurons and the structural integrity of the dopaminergic system in general may be intact in the alcohol exposed fish.

The dopaminergic system in the zebrafish brain is relatively well explored. For instance, dopaminergic neurons have been identified in subpallial regions (Tay et al., 2011) and in the periventricular nucleus of the posterior tuberculum of the zebrafish brain (basal diencephalon), a teleost dopaminergic system ascending to the subpallium 
equivalent to the mammalian striatum (Rink and Wullimann, 2001). The connectome, that is, both the outputs (target areas) and the inputs (neurons connecting to dopaminergic neurons) of the dopaminergic system involve numerous neurotransmitter systems in several areas of the zebrafish brain (Tay et al., 2011). It is thus possible that although the dopaminergic neurons themselves may be intact, the development of their connectome is altered by embryonic alcohol exposure, and this alteration may explain the unresponsive nature of dopaminergic neurons to social stimuli. The intact nature of dopaminergic neurons and the possibility of abnormal ascending inputs to these neurons are also supported indirectly by finding unaltered motivation of embryonic alcohol exposed zebrafish to seek and obtain food in an associative learning task (Fernandes et al., 2014). What exactly may underlie the altered dopaminergic response, how and where the connectome of these neurons may have been altered, which neurotransmitter systems and brain areas are involved, and what molecular mechanisms underlie these changes, will need to be investigated in the future.

\subsection{Prospects for modelling FASD in zebrafish}

Building an animal model of a human disease has an inherent paradox. In order for one to properly model a human disease, one has to understand the mechanisms of the disease. But most often the very reason why one would want to create an animal model is to investigate these mechanisms. The mild fetal alcohol spectrum disorder model with zebrafish described above suffers from the same problem. This model, as all animal models of human diseases, needs to meet three primary criteria: face, construct and predictive validity. The zebrafish FASD model arguably meets the face validity criterion. 
That is, the phenotypical features, or the appearance of symptoms, seen in zebrafish exposed to alcohol during embryonic development are similar to those seen in human FASD cases. For example, high alcohol doses applied for a prolonged period of time during development induce anatomical malformations resembling those seen in the severe FAS cases, and lower concentrations of alcohol employed for short period of time also lead to changes at the level of behavior found typical in milder FASD cases. Construct validity of the zebrafish model, however, has not been established. Although the causal factor, alcohol, underlying the changes observed in humans and zebrafish is the same, and although the dopaminergic system has been implicated in FASD (see similarities of findings in rodent and primate studies and the zebrafish results reviewed by Schneider et al., 2011), the mechanisms underlying the observed behavioral and neurochemical changes seen in zebrafish have not been systematically explored, and any biomarkers that may be similar across mammalian and fish species have not been established. Similarly, predictive validity, i.e. the question of whether the zebrafish model would respond to pharmacotherapy or other therapeutic approaches similarly to a human FASD patient will need to be assessed in the future. Clearly, the zebrafish FASD model is in its early building phase. Nevertheless, given the high translational relevance of the zebrafish established in multiple zebrafish models of human diseases (see review by Pickart and Klee, 2014), the promising results already obtained with the zebrafish FASD model, the sophisticated molecular biology, neurobiology and more recently behavioral tools developed for this species, coupled with the relatively low cost typical of zebrafish research, we hope that the zebrafish FASD model will significantly contribute 
to the understanding of the mechanisms, the diagnosis and the treatment of this life longdisease.

\section{The zebrafish is a promising tool. But there are many shortcomings of this species we need to overcome.}

This review sampled highly different zebrafish research areas. Common to all of them is the focus on the central nervous system and the use of behavioral methods. The advantage of behavioral tests over many other methods is that they allow the probing of the brain without having any preconceived notion of exactly what and where experimental manipulation induced modifications will occur. Indeed, behavioral tools have been successfully employed in a wide variety of research studies whose goal was to characterize or identify the experimentally induced functional change in the CNS, be it a genetic manipulation or a pharmacological agent or a neuroanatomical method such as lesioning. Although the past decade has seen a dramatic upsurge in studies of zebrafish employing behavioral methods (Kalueff et al., 2014), the number of behavioral tests developed for and the depth of our understanding of the behavioral features of the zebrafish is still dwarfed by studies on more traditional laboratory rodents, the house mouse and the rat (Kalueff et al., 2014; Sison et al., 2014). The behavioral bottle neck is a serious issue for zebrafish research, especially considering the dramatic development of molecular biology and the powerful recombinant DNA techniques developed for this species. These methods now allow unprecedented precision with which one can discover novel genes (forward genetics) or manipulate known genes (reverse genetics). Without proper behavioral tools, however, screening for mutation effects on brain function, or 
characterization of the effects of a targeted mutation of a gene expressed in the brain of zebrafish is difficult. Continued focus on the development of sophisticated behavioral paradigms and on detailed characterization of zebrafish behavior is warranted.

Another and perhaps equally important problem with zebrafish that we have already mentioned in the introduction concerns pharmacology. With the increasingly sophisticated recombinant DNA technologies, the use of compounds and drugs in research has been diminishing. This is understandable because recombinant DNA techniques offer unprecedented precision with which a biological system may be manipulated, its biochemical reactions altered, mapped and functionally characterized. In addition to being used as a research tool, recombinant DNA technologies now also offer therapeutic possibilities, such as the increasingly powerful gene therapy and/or recombinant protein-based methods. However, pharmacotherapy still represents an overwhelmingly dominant part of available therapeutic methods for countless human diseases. It is unlikely this will dramatically change in the near future. Pills will likely remain one of the most frequently used methods to treat people. Thus, psychopharmacology will remain an important field for translational or medical research and treatment development. Unfortunately, however, psychopharmacology of the zebrafish is a highly underexplored research area. The pharmacological characterization of compounds, small molecules with biological activity, is often completely lacking for the zebrafish. Although numerous studies, some of them reviewed above, have demonstrated evolutionary conservation of the effects of a range of drugs and compounds from fish to mammals, detailed pharmacological characterization of the compounds employed with zebrafish is rarely performed. Unlike for rodents, zebrafish researchers 
usually have little idea about ADME characteristics of the compound they are using. Receptor occupancy studies are also rarely available for zebrafish. The limited amount, or complete absence, of such information represents a significant bottleneck in zebrafish research.

\section{Overall outlook and summary}

This review focused on the use of the zebrafish in behavioral neuroscience and psychopharmacology research. Although the growth of studies with zebrafish in these research areas has been exponential, compared to the rat or the house mouse, the zebrafish is still a relative newcomer. Why should any experimenter use it then? There are numerous specific reasons, some of which we have discussed in this review, others have been emphasized elsewhere in the literature (Chen \& Ekker, 2004; Conant \& Wolfe, 2008; Gerlai, 2010; 2012; 2015; Kalueff et al., 2014; Levin et al., 2015; Morris, 2009; Patton \& Zon, 2001; Pickart \& Klee, 2014; Rihel \& Schier, 2012; Sison et al., 2006; Stewart et al., 2015a; 2014). From its practical advantages, low cost and simplicity, through the ease with which it may be manipulated using a variety of tools, to its translational relevance, many of the features of the zebrafish suggest that this small fish will continue to make waves in research. Although comparing advantages and disadvantages of particular research organisms is a useful exercise, perhaps an additional point should also be reemphasized here. Even if the zebrafish is inferior in some respects to other model organisms (e.g. consider its relative novice status and the bottleneck this may represent for certain research methods or approaches), adding it to research enhances 
translational relevance because it allows us to discover and focus on evolutionarily conserved, fundamental aspects of the studied phenomena (Gerlai, 2014).

\section{Acknowledgements and Author Contribution}

All authors provided critical comments on all aspects of the manuscript. RG organized the symposium upon which this review is based. Author SS was the primary contributor to the section on shoaling, JR the section on ASD, JGO on natural product screening, and RG on FASD. RG is supported by NSERC Canada (grant \# 311637). We wish to thank members of the Rihel, Ortiz, and Gerlai labs for critical discussion and comments. 


\section{References}

Abaid, N., Bartolini, T., Macri, S., Porfiri, M., 2012. Zebrafish responds differentially to a robotic fish of varying aspect ratio, tail beat frequency, noise, and color. Behavioral brain research 233, 545-553.

Ahrens, M.B., Li, J.M., Orger, M.B., Robson, D.N., Schier, A.F., Engert, F., Portugues, R., 2012. Brain-wide neuronal dynamics during motor adaptation in zebrafish. Nature 485, 471-477.

Al-Imari, L., Gerlai, R., 2008. Sight of conspecifics as reward in associative learning in zebrafish (Danio rerio). Behavioral brain research 189, 216-219.

Alvarado-Guzman, J.A., Gavillan-Suarez, J., Germosen-Robineau, L., 2009. TRAMIL ethnopharmacological survey: knowledge distribution of medicinal plant use in the southeast region of Puerto Rico. Puerto Rico health sciences journal 28, 329339.

American Psychiatric Association., 2013. Diagnostic and statistical manual of mental disorders 5th ed. Arlington, VA: American Psychiatric Publishing.

Amsterdam, A., Hopkins, N., 2006. Mutagenesis strategies in zebrafish for identifying genes involved in development and disease. Trends in genetics: TIG 22, 473-478.

Appelbaum, L., Wang, G., Yokogawa, T., Skariah, G.M., Smith, S.J., Mourrain, P., Mignot, E., 2010. Circadian and homeostatic regulation of structural synaptic plasticity in hypocretin neurons. Neuron 68, 87-98.

Arenzana, F.J., Carvan, M.J., 3rd, Aijon, J., Sanchez-Gonzalez, R., Arevalo, R., Porteros, A., 2006. Teratogenic effects of ethanol exposure on zebrafish visual system development. Neurotoxicology and teratology 28, 342-348. 
Baraban, S.C., Dinday, M.T., Hortopan, G.A., 2013. Drug screening in Scn1a zebrafish mutant identifies clemizole as a potential Dravet syndrome treatment. Nature communications 4, 2410.

Baraban, S.C., Taylor, M.R., Castro, P.A., Baier, H., 2005. Pentylenetetrazole induced changes in zebrafish behavior, neural activity and c-fos expression. Neuroscience 131, 759-768.

Barba-Escobedo, P.A., Gould, G.G., 2012. Visual social preferences of lone zebrafish in a novel environment: strain and anxiolytic effects. Genes, brain, and behavior 11, 366-373.

Battle, H.I., Mcleese, L., 1954. Effects of Urethane (Ethyl Carbamate) on Mitochondria of Zebrafish Blastomeres. Anatomical Record 120, 709-709

Bencan, Z., Sledge, D., Levin, E.D., 2009. Buspirone, chlordiazepoxide and diazepam effects in a zebrafish model of anxiety. Pharmacology, biochemistry, and behavior $94,75-80$.

Bernier, R., Golzio, C., Xiong, B., Stessman, H.A., Coe, B.P., Penn, O., et al., 2014. Disruptive CHD8 mutations define a subtype of autism early in development. Cell $158,263-276$.

Bilotta, J., Barnett, J.A., Hancock, L., Saszik, S., 2004. Ethanol exposure alters zebrafish development: a novel model of fetal alcohol syndrome. Neurotoxicology and teratology $26,737-743$.

Blechman, J., Amir-Zilberstein, L., Gutnick, A., Ben-Dor, S., Levkowitz, G., 2011. The metabolic regulator PGC-1alpha directly controls the expression of the 
hypothalamic neuropeptide oxytocin. The Journal of neuroscience: the official journal of the Society for Neuroscience 31, 14835-14840.

Bohni, N., Cordero-Maldonado, M.L., Maes, J., Siverio-Mota, D., Marcourt, L., Munck, S., et al., 2013. Integration of Microfractionation, qNMR and zebrafish screening for the in vivo bioassay-guided isolation and quantitative bioactivity analysis of natural products. PloS one 8, e64006.

Brillatz, T., Ferreira Queiroz, E., Marcourt, L., Vougogiannopoulou, K., Jacmin, M., Crawford, A.D., Skaltsounis, L., Wolfender, J.L., 2016. Bioguided isolation of anticonvulsant principles from Helleborus cyclophyllus using the zebrafish epilepsy model. Planta medica 81, S1-s381.

Budick, S.A., O'Malley, D.M., 2000. Locomotor repertoire of the larval zebrafish: swimming, turning and prey capture. The Journal of experimental biology 203, 2565-2579.

Burgess, H.A., Granato, M., 2007. Sensorimotor gating in larval zebrafish. The Journal of neuroscience: the official journal of the Society for Neuroscience 27, 4984-4994.

Buske, C., Gerlai, R., 2011. Shoaling develops with age in Zebrafish (Danio rerio). Progress in neuro-psychopharmacology \& biological psychiatry 35, 1409-1415.

Buske, C., Gerlai, R., 2012. Maturation of shoaling behavior is accompanied by changes in the dopaminergic and serotoninergic systems in zebrafish. Developmental psychobiology 54, 28-35.

Buske, C., Gerlai, R., 2014. Diving deeper into Zebrafish development of social behavior: analyzing high resolution data. Journal of neuroscience methods 234, 66-72. 
Butail, S., Polverino, G., Phamduy, P., Del Sette, F., Porfiri, M., 2014. Influence of robotic shoal size, configuration, and activity on zebrafish behavior in a freeswimming environment. Behavioral brain research 275, 269-280.

Carvan, M.J., 3rd, Loucks, E., Weber, D.N., Williams, F.E., 2004. Ethanol effects on the developing zebrafish: neurobehavior and skeletal morphogenesis. Neurotoxicology and teratology 26, 757-768.

Challal, S., Buenafe, O.E., Queiroz, E.F., Maljevic, S., Marcourt, L., Bock, M., et al., 2014. Zebrafish bioassay-guided microfractionation identifies anticonvulsant steroid glycosides from the Philippine medicinal plant Solanum torvum. ACS chemical neuroscience 5, 993-1004.

Chatterjee, D., Gerlai, R., 2009. High precision liquid chromatography analysis of dopaminergic and serotoninergic responses to acute alcohol exposure in zebrafish. Behavioral brain research 200, 208-213.

Chen, E., Ekker, S.C., 2004. Zebrafish as a genomics research model. Current pharmaceutical biotechnology 5, 409-413.

Chen, S., Chiu, C.N., McArthur, K.L., Fetcho, J.R., Prober, D.A., 2016. TRP channel mediated neuronal activation and ablation in freely behaving zebrafish. Nature methods $13,147-150$.

Christensen, D.L., Baio, J., Van Naarden Braun, K., Bilder, D., Charles, J., Constantino, J.N., et al., 2016. Prevalence and Characteristics of Autism Spectrum Disorder Among Children Aged 8 Years--Autism and Developmental Disabilities Monitoring Network, 11 Sites, United States, 2012. Morbidity and mortality weekly report. Surveillance summaries (Washington, D.C.: 2002) 65, 1-23. 
Coe, T.S., Soffker, M.K., Filby, A.L., Hodgson, D., Tyler, C.R., 2010. Impacts of early life exposure to estrogen on subsequent breeding behavior and reproductive success in zebrafish. Environmental science \& technology 44, 6481-6487.

Conant, G.C., Wolfe, K.H., 2008. Turning a hobby into a job: how duplicated genes find new functions. Nature reviews. Genetics 9, 938-950.

Cowden, J., Padnos, B., Hunter, D., MacPhail, R., Jensen, K., Padilla, S., 2012. Developmental exposure to valproate and ethanol alters locomotor activity and retino-tectal projection area in zebrafish embryos. Reproductive toxicology (Elmsford, N.Y.) 33, 165-173.

Cragg, G.M., Newman, D.J., 2013. Natural products: a continuing source of novel drug leads. Biochimica et biophysica acta 1830, 3670-3695.

Crawford, B.D., Po, M.D., Saranyan, P.V., Forsberg, D., Schulz, R., Pilgrim, D.B., 2014. Mmp25beta facilitates elongation of sensory neurons during zebrafish development. Genesis (New York, N.Y.: 2000) 52, 833-848.

Dahlbom, S.J., Backstrom, T., Lundstedt-Enkel, K., Winberg, S., 2012. Aggression and monoamines: effects of sex and social rank in zebrafish (Danio rerio). Behavioral brain research 228, 333-338.

Dani, V.S., Chang, Q., Maffei, A., Turrigiano, G.G., Jaenisch, R., Nelson, S.B., 2005. Reduced cortical activity due to a shift in the balance between excitation and inhibition in a mouse model of Rett syndrome. Proceedings of the National Academy of Sciences of the United States of American 102, 12560-12565. 
De Rubeis, S., He, X., Goldberg, A.P., Poultney, C.S., Samocha, K., Cicek, A.E., et al., 2014. Synaptic, transcriptional and chromatin genes disrupted in autism. Nature $515,209-215$.

Del Valle-Mojica, L.M., Ayala-Marin, Y.M., Ortiz-Sanchez, C.M., Torres-Hernandez, B.A., Abdalla-Mukhaimer, S., Ortiz, J.G., 2011. Selective Interactions of Valeriana officinalis Extracts and Valerenic Acid with $[\mathrm{H}]$ Glutamate Binding to Rat Synaptic Membranes. Evidence-based complementary and alternative medicine: eCAM 2011, 403591.

Dinday, M.T., Baraban, S.C., 2015. Large-Scale Phenotype-Based Antiepileptic Drug Screening in a Zebrafish Model of Dravet Syndrome (1,2,3). eNeuro 2.

Dreosti, E., Lopes, G., Kampff, A.R., Wilson, S.W., 2015. Development of social behavior in young zebrafish. Frontiers in neural circuits 9, 39.

Eisen, J.S., Smith, J.C., 2008. Controlling morpholino experiments: don't stop making antisense. Development (Cambridge, England) 135, 1735-1743.

Ellegood, J., Anagnostou, E., Babineau, B.A., Crawley, J.N., Lin, L., Genestine, M., et al., 2015. Clustering autism: using neuroanatomical differences in 26 mouse models to gain insight into the heterogeneity. Molecular psychiatry 20, 118-125.

Ellis, L.D., Seibert, J., Soanes, K.H., 2012. Distinct models of induced hyperactivity in zebrafish larvae. Brain research 1449, 46-59.

Elsen, G.E., Choi, L.Y., Prince, V.E., Ho, R.K., 2009. The autism susceptibility gene met regulates zebrafish cerebellar development and facial motor neuron migration. Developmental biology 335, 78-92. 
Emran, F., Rihel, J., Adolph, A.R., Wong, K.Y., Kraves, S., Dowling, J.E., 2007. OFF ganglion cells cannot drive the optokinetic reflex in zebrafish. Proceedings of the National Academy of Sciences of the United States of America 104, 1912619131.

Engeszer, R.E., Patterson, L.B., Rao, A.A., Parichy, D.M., 2007. Zebrafish in the wild: a review of natural history and new notes from the field. Zebrafish 4, 21-40.

Engeszer, R.E., Ryan, M.J., Parichy, D.M., 2004. Learned social preference in zebrafish. Current biology: CB 14, 881-884.

Ey, E., Leblond, C.S., Bourgeron, T., 2011. Behavioral profiles of mouse models for autism spectrum disorders. Autism research: official journal of the International Society for Autism Research 4, 5-16.

Fadool, J.M., Dowling, J.E., 2008. Zebrafish: a model system for the study of eye genetics. Progress in retinal and eye research 27, 89-110.

Fernandes, Y., Gerlai, R., 2009. Long-term behavioral changes in response to early developmental exposure to ethanol in zebrafish. Alcoholism, clinical and experimental research 33, 601-609.

Fernandes, Y., Rampersad, M., Gerlai, R., 2015a. Embryonic alcohol exposure impairs the dopaminergic system and social behavioral responses in adult zebrafish. The international journal of neuropsychopharmacology/official scientific journal of the Collegium Internationale Neuropsychopharmacologicum (CINP) 18.

Fernandes, Y., Rampersad, M., Gerlai, R., 2015b. Impairment of social behavior persists two years after embryonic alcohol exposure in zebrafish: A model of fetal alcohol spectrum disorders. Behavioral brain research 292, 102-108. 
Fernandes, Y., Tran, S., Abraham, E., Gerlai, R., 2014. Embryonic alcohol exposure impairs associative learning performance in adult zebrafish. Behavioral brain research 265, 181-187.

Field, H.A., Kelley, K.A., Martell, L., Goldstein, A.M., Serluca, F.C., 2009. Analysis of gastrointestinal physiology using a novel intestinal transit assay in zebrafish. Neurogastroenterology and motility: the official journal of the European Gastrointestinal Motility Society 21, 304-312.

Fontaine, E., Lentink, D., Kranenbarg, S., Muller, U.K., van Leeuwen, J.L., Barr, A.H., Burdick, J.W., 2008. Automated visual tracking for studying the ontogeny of zebrafish swimming. The Journal of experimental biology 211, 1305-1316.

Gauthier, J., Champagne, N., Lafreniere, R.G., Xiong, L., Spiegelman, D., Brustein, E., et al., 2010. De novo mutations in the gene encoding the synaptic scaffolding protein SHANK3 in patients ascertained for schizophrenia. Proceedings of the National Academy of Sciences of the United States of America 107, 7863-7868.

Gebauer, D.L., Pagnussat, N., Piato, A.L., Schaefer, I.C., Bonan, C.D., Lara, D.R., 2011. Effects of anxiolytics in zebrafish: similarities and differences between benzodiazepines, buspirone and ethanol. Pharmacology, biochemistry, and behavior $99,480-486$.

Gerlach, G., Hodgins-Davis, A., Avolio, C., Schunter, C., 2008. Kin recognition in zebrafish: a 24-hour window for olfactory imprinting. Proceedings. Biological sciences / The Royal Society 275, 2165-2170.

Gerlai, R. 2002. Phenomics: Fiction or the Future? Trends Neurosci. 25: 506-509. 
Gerlai, R., 2010. High-throughput behavioral screens: the first step towards finding genes involved in vertebrate brain function using zebrafish. Molecules (Basel, Switzerland) 15, 2609-2622.

Gerlai, R., 2012. Using zebrafish to unravel the genetics of complex brain disorders. Current topics in behavioral neurosciences 12, 3-24.

Gerlai, R., 2014. Fish in behavior research: unique tools with a great promise! Journal of neuroscience methods $234,54-58$.

Gerlai R, 2014b. Zebrafish Phenomics: Behavioral Screens and Phenotyping of Mutagenized Fish. Current Opin Behav Sci 2: 21-27.

Gerlai R., 2015. Embryonic alcohol exposure: Towards the development of a zebrafish model of fetal alcohol spectrum disorders. Developmental Psychobiology 57: 787-798.

Geschwind, D.H., 2009. Advances in autism. Annual review of medicine 60, 367-380.

Giacomini, A.C., Abreu, M.S., Giacomini, L.V., Siebel, A.M., Zimerman, F.F., Rambo, C.L., et al., 2016. Fluoxetine and diazepam acutely modulate stress inducedbehavior. Behavioral brain research 296, 301-310.

Golzio, C., Willer, J., Talkowski, M.E., Oh, E.C., Taniguchi, Y., Jacquemont, S., et al., 2012. KCTD13 is a major driver of mirrored neuroanatomical phenotypes of the 16p11.2 copy number variant. Nature 485, 363-367.

Gómez-Laplaza, L.M., Gerlai, R., 2011. Spontaneous discrimination of small quantities: shoaling preferences in angelfish (Pterophyllum scalare). Animal cognition 14, $565-574$. 
Greenbaum, R.L., Stevens, S.A., Nash, K., Koren, G., Rovet, J., 2009. Social cognitive and emotion processing abilities of children with fetal alcohol spectrum disorders: a comparison with attention deficit hyperactivity disorder. Alcoholism, clinical and experimental research 33, 1656-1670.

Gregson, J.N.S., Burt de Perera, T., 2007. Shoaling in eyed and blind morphs of the characin Astyanax fasciatus under light and dark conditions. Journal of fish biology 70, 1615-1619.

Haaz, S., Fontaine, K.R., Cutter, G., Limdi, N., Perumean-Chaney, S., Allison, D.B., 2006. Citrus aurantium and synephrine alkaloids in the treatment of overweight and obesity: an update. Obesity reviews: an official journal of the International Association for the Study of Obesity 7, 79-88.

Henry, J.D., von Hippel, W., Molenberghs, P., Lee, T., Sachdev, P.S., 2016. Clinical assessment of social cognitive function in neurological disorders. Nat Rev Neurol $12,28-39$.

Hernandez, L., Munoz, R.A., Miro, G., Martinez, M., Silva-Parra, J., Chavez, P.I., 1984. Use of medicinal plants by ambulatory patients in Puerto Rico. American journal of hospital pharmacy 41, 2060-2064.

Hinz, R. C., \& de Polavieja, G. G. 2017. Ontogeny of collective behavior reveals a simple attraction rule. Proc Natl Acad Sci U S A, 114(9), 2295-2300.

Hisaoka K.K., Hopper A.F., 1957. Some effects of barbituric and diethylbarbituric acid on the development of the zebra fish, Brachydanio rerio. Anat Rec. 129, 297-307. Hoffman, E.J., Turner, K.J., Fernandez, J.M., Cifuentes, D., Ghosh, M., Ijaz, S., Jain, R.A., Kubo, F., Bill, B.R., Baier, H., Granato, M., Barresi, M.J., Wilson, S.W., 
Rihel, J., State, M.W., Giraldez, A.J., 2016. Estrogens Suppress a Behavioral Phenotype in Zebrafish Mutants of the Autism Risk Gene, CNTNAP2. Neuron $89,725-733$.

Howe, K., Clark, M.D., Torroja, C.F., Torrance, J., Berthelot, C., Muffato, M., et al., 2013. The zebrafish reference genome sequence and its relationship to the human genome. Nature 496, 498-503.

Iossifov, I., O’Roak, B.J., Sanders, S.J., Ronemus, M., Krumm, N., Levy, D., et al., 2014. The contribution of de novo coding mutations to autism spectrum disorder. Nature $515,216-221$.

Jao, L.E., Wente, S.R., Chen, W., 2013. Efficient multiplex biallelic zebrafish genome editing using a CRISPR nuclease system. Proceedings of the National Academy of Sciences of the United States of America 110, 13904-13909.

Jones, L.J., Norton, W.H., 2015. Using zebrafish to uncover the genetic and neural basis of aggression, a frequent comorbid symptom of psychiatric disorders. Behavioral brain research 276, 171-180.

Kalueff, A.V., Stewart, A.M., Gerlai, R., 2014. Zebrafish as an emerging model for studying complex brain disorders. Trends in pharmacological sciences 35, 63-75.

Kawakami, K., Asakawa, K., Hibi, M., Itoh, M., Muto, A., Wada, H., 2016. Gal4 Driver Transgenic Zebrafish: Powerful Tools to Study Developmental Biology, Organogenesis, and Neuroscience. Advances in genetics 95, 65-87.

Kawashima, T., Zwart, M.F., Yang, C.T., Mensh, B.D., Ahrens, M.B., 2016. The Serotonergic System Tracks the Outcomes of Actions to Mediate Short-Term Motor Learning. Cell 167, 933-946.e920. 
Keck, V.A., Edgerton, D.S., Hajizadeh, S., Swift, L.L., Dupont, W.D., Lawrence, C., Boyd, K.L., 2015. Effects of Habitat Complexity on Pair-Housed Zebrafish. Journal of the American Association for Laboratory Animal Science: JAALAS $54,378-383$.

Kimmel, C.B., Ballard, W.W., Kimmel, S.R., Ullmann, B., Schilling, T.F., 1995. Stages of embryonic development of the zebrafish. Developmental dynamics: an official publication of the American Association of Anatomists 203, 253-310.

King, I.F., Yandava, C.N., Mabb, A.M., Hsiao, J.S., Huang, H.S., Pearson, B.L., Calabrese, J.M., Starmer, J., Parker, J.S., Magnuson, T., Chamberlain, S.J., Philpot, B.D., Zylka, M.J., 2013. Topoisomerases facilitate transcription of long genes linked to autism. Nature 501, 58-62.

Kitevski B., Pyron, M. 2003. Female zebrafish (Danio rerio) do not prefer mutant longfin males. Journal of freshwater ecology 18, 501-502.

Klafki, H.W., Staufenbiel, M., Kornhuber, J., Wiltfang, J., 2006. Therapeutic approaches to Alzheimer's disease. Brain: a journal of neurology 129, 2840-2855.

Kok, F.O., Shin, M., Ni, C.W., Gupta, A., Grosse, A.S., van Impel, A., et al., 2015. Reverse genetic screening reveals poor correlation between morpholino-induced and mutant phenotypes in zebrafish. Developmental cell 32, 97-108.

Kozol, R.A., Abrams, A.J., James, D.M., Buglo, E., Yan, Q., Dallman, J.E., 2016. Function Over Form: Modeling Groups of Inherited Neurological Conditions in Zebrafish. Frontiers in molecular neuroscience 9, 55.

Kozol, R.A., Cukier, H.N., Zou, B., Mayo, V., De Rubeis, S., Cai, G., et al., 2015. Two knockdown models of the autism genes SYNGAP1 and SHANK3 in zebrafish 
produce similar behavioral phenotypes associated with embryonic disruptions of brain morphogenesis. Human molecular genetics 24, 4006-4023.

Krause, J., Ruxton, G.D., 2002. Living in groups. Oxford, Oxford University Press, pp 205.

Krishnan, A., Zhang, R., Yao, V., Theesfeld, C.L., Wong, A.K., Tadych, A., et al., 2016. Genome-wide prediction and functional characterization of the genetic basis of autism spectrum disorder. Nature neuroscience 19, 1454-1462.

Ladu, F., Bartolini, T., Panitz, S.G., Chiarotti, F., Butail, S., Macri, S., Porfiri, M., 2015. Live predators, robots, and computer-animated images elicit differential avoidance responses in zebrafish. Zebrafish 12, 205-214.

Lai, M.C., Lombardo, M.V., Baron-Cohen, S., 2014. Autism. Lancet (London, England) 383, 896-910.

Larson, E.T., O’Malley, D.M., Melloni, R.H., Jr., 2006. Aggression and vasotocin are associated with dominant-subordinate relationships in zebrafish. Behavioral brain research 167, 94-102.

Lawson, N.D., 2016. Reverse Genetics in Zebrafish: Mutants, Morphants, and Moving Forward. Trends in cell biology 26, 77-79.

Lazarowski, A., Czornyj, L., Lubienieki, F., Girardi, E., Vazquez, S., D’Giano, C., 2007. $\mathrm{ABC}$ transporters during epilepsy and mechanisms underlying multidrug resistance in refractory epilepsy. Epilepsia 48 Suppl 5, 140-149.

Lee, Y., Kim, D., Kim, Y.H., Lee, H., Lee, C.J., 2010. Improvement of pentylenetetrazolinduced learning deficits by valproic acid in the adult zebrafish. European journal of pharmacology 643, 225-231. 
Lekven, A.C., Helde, K.A., Thorpe, C.J., Rooke, R., Moon, R.T., 2000. Reverse genetics in zebrafish. Physiological genomics 2, 37-48.

Levin, E.D., Kalueff, A.V., Gerlai, R.T., 2015. Perspectives on zebrafish neurobehavioral pharmacology. Pharmacology, biochemistry, and behavior 139 Pt B, 93.

Mahabir, S., Chatterjee, D., Buske, C., Gerlai, R., 2013. Maturation of shoaling in two zebrafish strains: a behavioral and neurochemical analysis. Behavioral brain research $247,1-8$.

Mahabir, S., Chatterjee, D., Gerlai, R., 2014. Strain dependent neurochemical changes induced by embryonic alcohol exposure in zebrafish. Neurotoxicology and teratology $41,1-7$.

Mathias, J.R., Zhang, Z., Saxena, M.T., Mumm, J.S., 2014. Enhanced cell-specific ablation in zebrafish using a triple mutant of Escherichia coli nitroreductase. Zebrafish 11, 85-97.

Mattson, S.N., Crocker, N., Nguyen, T.T., 2011. Fetal alcohol spectrum disorders: neuropsychological and behavioral features. Neuropsychology review 21, 81-101.

Maximino, C., de Brito, T.M., da Silva Batista, A.W., Herculano, A.M., Morato, S., Gouveia, A., Jr., 2010. Measuring anxiety in zebrafish: a critical review. Behavioral brain research 214, 157-171.

May, P.A., Gossage, J.P., Kalberg, W.O., Robinson, L.K., Buckley, D., Manning, M., Hoyme, H.E., 2009. Prevalence and epidemiologic characteristics of FASD from various research methods with an emphasis on recent in-school studies. Developmental disabilities research reviews 15, 176-192. 
McCammon, J.M., Sive, H., 2015. Challenges in understanding psychiatric disorders and developing therapeutics: a role for zebrafish. Disease models \& mechanisms 8 , 647-656.

Miller, N., Gerlai, R., 2007. Quantification of shoaling behavior in zebrafish (Danio rerio). Behavioral brain research 184, 157-166.

Miller, N., Gerlai, R., 2011a. Redefining membership in animal groups. Behavior research methods 43, 964-970.

Miller, N.Y., Gerlai, R., 2011b. Shoaling in zebrafish: what we don't know. Reviews in the neurosciences $22,17-25$.

Miller, N., Gerlai, R., 2012. From schooling to shoaling: patterns of collective motion in zebrafish (Danio rerio). PloS one 7, e48865.

Miller, N., Greene, K., Dydinski, A., Gerlai, R., 2013. Effects of nicotine and alcohol on zebrafish (Danio rerio) shoaling. Behavioral brain research 240, 192-196.

Miller, N.Y., Gerlai, R., 2008. Oscillations in shoal cohesion in zebrafish (Danio rerio). Behavioral brain research 193, 148-151.

Moretz, J.A., Martins, E.P., Robison, B.D., 2007. The effects of early and adult social environment on zebrafish (Danio rerio) behavior. Environmental biology of fishes 80, 91-100.

Morris, J.A., 2009. Zebrafish: a model system to examine the neurodevelopmental basis of schizophrenia. Progress in brain research 179, 97-106.

Nasevicius, A., Ekker, S.C., 2000. Effective targeted gene 'knockdown' in zebrafish. Nature genetics 26, 216-220. 
Nash, K., Rovet, J., Greenbaum, R., Fantus, E., Nulman, I., Koren, G., 2006. Identifying the behavioral phenotype in Fetal Alcohol Spectrum Disorder: sensitivity, specificity and screening potential. Archives of women's mental health 9, 181186.

Nash, K., Sheard, E., Rovet, J., Koren, G., 2008. Understanding fetal alcohol spectrum disorders (FASDs): toward identification of a behavioral phenotype. The Scientific World Journal 8, 873-882.

National Institute of Health: Office of Dietary Supplements, 2013. Valerian-Fact Sheet for Health Professional. [Online]. Retrieved Jan, 2017 from http://ods.od.nih.gov/pdf/factsheets/Valerian-HealthProfessional.pdf

Newman, D.J., Cragg, G.M., 2012. Natural products as sources of new drugs over the 30 years from 1981 to 2010 . Journal of natural products $75,311-335$.

Norton, W.H., 2013. Toward developmental models of psychiatric disorders in zebrafish. Frontiers in neural circuits 7, 79.

Oksenberg, N., Stevison, L., Wall, J.D., Ahituv, N., 2013. Function and regulation of AUTS2, a gene implicated in autism and human evolution. PLoS genetics 9, e1003221.

Panula, P., Chen, Y.C., Priyadarshini, M., Kudo, H., Semenova, S., Sundvik, M., Sallinen, V., 2010. The comparative neuroanatomy and neurochemistry of zebrafish CNS systems of relevance to human neuropsychiatric diseases. Neurobiology of disease 40, 46-57. 
Parikshak, N.N., Luo, R., Zhang, A., Won, H., Lowe, J.K., Chandran, V., Horvath, S., Geschwind, D.H., 2013. Integrative functional genomic analyses implicate specific molecular pathways and circuits in autism. Cell 155, 1008-1021.

Partridge, B.L., Pitcher, T.J., 1980. The sensory basis of fish schools: relative roles of lateral line and vision. Journal of comparative physiology 135, 315-325.

Patten, A. R., Fontaine, C. J., \& Christie, B. R., 2014. A comparison of the different animal models of fetal alcohol spectrum disorders and their use in studying complex behaviors. Front Pediatr. 2, 93 doi: 10.3389/fped.2014.00093

Patton, E.E., Zon, L.I., 2001. The art and design of genetic screens: zebrafish. Nature reviews. Genetics 2, 956-966.

Pavlidis, M., Sundvik, M., Chen, Y.C., Panula, P., 2011. Adaptive changes in zebrafish brain in dominant-subordinate behavioral context. Behavioral brain research 225, $529-537$.

Penagarikano, O., 2017. Oxytocin in Animal Models of Autism Spectrum Disorder. Developmental Neurobiology 77: 202-213.

Penagarikano, O., Abrahams, B.S., Herman, E.I., Winden, K.D., Gdalyahu, A., Dong, H., Sonnenblick, L.I., Gruver, R., Almajano, J., Bragin, A., Golshani, P., Trachtenberg, J.T., Peles, E., Geschwind, D.H., 2011. Absence of CNTNAP2 leads to epilepsy, neuronal migration abnormalities, and core autism-related deficits. Cell 147, 235-246.

Pickart, M.A., Klee, E.W., 2014. Zebrafish approaches enhance the translational research tackle box. Translational research: the journal of laboratory and clinical medicine $163,65-78$. 
Pietri, T., Roman, A.C., Guyon, N., Romano, S.A., Washbourne, P., Moens, C.B., de Polavieja, G.G., Sumbre, G., 2013. The first mecp2-null zebrafish model shows altered motor behaviors. Frontiers in neural circuits 7, 118.

Pimenta, F.C., Alves, M.F., Pimenta, M.B., Melo, S.A., de Almeida, A.A., Leite, J.R., Pordeus, L.C., Diniz Mde, F., de Almeida, R.N., 2016. Anxiolytic Effect of Citrus aurantium L. on Patients with Chronic Myeloid Leukemia. Phytotherapy research: PTR 30, 613-617.

Picher, T.J., 1993. Functions of shoaling behavior in teleosts. In: Pitcher TJ, Ed., Behavior of Teleost Fishes. London, Chapman \& Hall, pp. 294-337.

Poelmans, G., Franke, B., Pauls, D.L., Glennon, J.C., Buitelaar, J.K., 2013. AKAPs integrate genetic findings for autism spectrum disorders. Translational psychiatry 3, e270.

Poewe, W., 2009. Treatments for Parkinson disease--past achievements and current clinical needs. Neurology 72, S65-73.

Portugues, R., Severi, K.E., Wyart, C., Ahrens, M.B., 2013. Optogenetics in a transparent animal: circuit function in the larval zebrafish. Current opinion in neurobiology 23, 119-126.

Qin, M., Wong, A., Seguin, D., Gerlai, R., 2014. Induction of social behavior in zebrafish: live versus computer animated fish as stimuli. Zebrafish 11, 185-197.

Randlett, O., Wee, C.L., Naumann, E.A., Nnaemeka, O., Schoppik, D., Fitzgerald, J.E., Portugues, R., Lacoste, A.M., Riegler, C., Engert, F., Schier, A.F., 2015. Wholebrain activity mapping onto a zebrafish brain atlas. Nature methods 12 , 10391046. 
Rihel, J., Prober, D.A., Schier, A.F., 2010. Monitoring sleep and arousal in zebrafish. Methods in cell biology 100, 281-294.

Rihel, J., Schier, A.F., 2012. Behavioral screening for neuroactive drugs in zebrafish. Developmental neurobiology 72, 373-385.

Rink, E., Wullimann, M.F., 2001. The teleostean (zebrafish) dopaminergic system ascending to the subpallium (striatum) is located in the basal diencephalon (posterior tuberculum). Brain research 889, 316-330.

Roeser, T., Baier, H., 2003. Visuomotor behaviors in larval zebrafish after GFP-guided laser ablation of the optic tectum. The Journal of neuroscience: the official journal of the Society for Neuroscience 23, 3726-3734.

Rosa-Falero, C., Torres-Rodriguez, S., Jordan, C., Licier, R., Santiago, Y., Toledo, Z., Santiago, M., Serrano, K., Sosa, J., Ortiz, J.G., 2014. Citrus aurantium increases seizure latency to PTZ induced seizures in zebrafish thru NMDA and mGluR's I and II. Frontiers in pharmacology 5, 284.

Rossi, A., Kontarakis, Z., Gerri, C., Nolte, H., Holper, S., Kruger, M., Stainier, D.Y., 2015. Genetic compensation induced by deleterious mutations but not gene knockdowns. Nature 524, 230-233.

Ruhl, N., McRobert, S.P., 2005. The effect of sex and shoal size on shoaling behavior in Danio rerio. Journal of freshwater ecology 67, 1318-1326.

Ruhl, N., McRobert, S.P., Currie, W.J., 2009. Shoaling preferences and the effects of sex ratio on spawning and aggression in small laboratory populations of zebrafish (Danio rerio). Lab animal 38, 264-269. 
Saif, M., Chatterjee, D., Buske, C., Gerlai, R., 2013. Sight of conspecific images induces changes in neurochemistry in zebrafish. Behavioral brain research 243, 294-299.

Saili, K.S., Corvi, M.M., Weber, D.N., Patel, A.U., Das, S.R., Przybyla, J., Anderson, K.A., Tanguay, R.L., 2012. Neurodevelopmental low-dose bisphenol A exposure leads to early life-stage hyperactivity and learning deficits in adult zebrafish. Toxicology 291, 83-92.

Saint-Amant, L., Drapeau, P., 1998. Time course of the development of motor behaviors in the zebrafish embryo. Journal of neurobiology 37, 622-632.

Sampson, P.D., Streissguth, A.P., Bookstein, F.L., Little, R.E., Clarren, S.K., Dehaene, P., Hanson, J.W., Graham, J.M., Jr., 1997. Incidence of fetal alcohol syndrome and prevalence of alcohol-related neurodevelopmental disorder. Teratology 56, 317-326.

Sanders, S.J., Ercan-Sencicek, A.G., Hus, V., Luo, R., Murtha, M.T., Moreno-De-Luca, et al., 2011. Multiple recurrent de novo CNVs, including duplications of the 7q11.23 Williams syndrome region, are strongly associated with autism. Neuron $70,863-885$.

Satou, C., Kimura, Y., Hirata, H., Suster, M.L., Kawakami, K., Higashijima, S., 2013. Transgenic tools to characterize neuronal properties of discrete populations of zebrafish neurons. Development (Cambridge, England) 140, 3927-3931.

Saverino, C., Gerlai, R., 2008. The social zebrafish: behavioral responses to conspecific, heterospecific, and computer animated fish. Behavioral brain research 191, 77-87.

Savio, L.E., Vuaden, F.C., Piato, A.L., Bonan, C.D., Wyse, A.T., 2012. Behavioral changes induced by long-term proline exposure are reversed by antipsychotics in 
zebrafish. Progress in neuro-psychopharmacology \& biological psychiatry 36, 258-263.

Scerbina, T., Chatterjee, D., Gerlai, R., 2012. Dopamine receptor antagonism disrupts social preference in zebrafish: a strain comparison study. Amino acids 43, 20592072.

Schaefer, I.C., Siebel, A.M., Piato, A.L., Bonan, C.D., Vianna, M.R., Lara, D.R., 2015. The side-by-side exploratory test: a simple automated protocol for the evaluation of adult zebrafish behavior simultaneously with social interaction. Behavioral pharmacology 26, 691-696.

Schneider, M.L., Moore, C.F., Adkins, M.M., 2011. The effects of prenatal alcohol exposure on behavior: rodent and primate studies. Neuropsychology review 21, 186-203.

Seguin, D., Gerlai, R., 2017. Zebrafish prefer larger to smaller shoals: analysis of quantity estimation in a genetically tractable model organism. Animal Cognition doi: 10.1007/s10071-017-1102-x (in press).

Seguin, D., Shams, S., Gerlai, R., 2016. Behavioral Responses to Novelty or to a Predator Stimulus Are Not Altered in Adult Zebrafish by Early Embryonic Alcohol Exposure. Alcoholism, clinical and experimental research 40, 2667-2675.

Severi, K.E., Portugues, R., Marques, J.C., O'Malley, D.M., Orger, M.B., Engert, F., 2014. Neural control and modulation of swimming speed in the larval zebrafish. Neuron 83, 692-707.

Shamay-Ramot, A., Khermesh, K., Porath, H.T., Barak, M., Pinto, Y., Wachtel, C., Zilberberg, A., Lerer-Goldshtein, T., Efroni, S., Levanon, E.Y., Appelbaum, L., 
2015. Fmrp Interacts with Adar and Regulates RNA Editing, Synaptic Density and Locomotor Activity in Zebrafish. PLoS genetics 11, e1005702.

Shams, S., Chatterjee, D., Gerlai, R., 2015. Chronic social isolation affects thigmotaxis and whole-brain serotonin levels in adult zebrafish. Behavioral brain research 292, 283-287.

Shams, S., Gerlai, R., 2016. Pharmacological manipulation of shoaling behavior in zebrafish. Current Psychopharmacology 5, 180-193.

Shelton, R.C., Osuntokun, O., Heinloth, A.N., Corya, S.A., 2010. Therapeutic options for treatment-resistant depression. CNS drugs 24, 131-161.

Sison, M., Cawker, J., Buske, C., Gerlai, R., 2006. Fishing for genes influencing vertebrate behavior: zebrafish making headway. Lab animal 35, 33-39.

Speedie, N., Gerlai, R., 2008. Alarm substance induced behavioral responses in zebrafish (Danio rerio). Behavioral brain research 188, 168-177.

Spence, R., Gerlach, G., Lawrence, C., Smith, C., 2008. The behavior and ecology of the zebrafish, Danio rerio. Biological reviews of the Cambridge Philosophical Society $83,13-34$.

Stevens, S.A., Dudek, J., Nash, K., Koren, G., Rovet, J., 2015. Social Perspective Taking and Empathy in Children with Fetal Alcohol Spectrum Disorders. Journal of the International Neuropsychological Society: JINS 21, 74-84.

Stewart, A.M., Braubach, O., Spitsbergen, J., Gerlai, R., Kalueff, A.V., 2014. Zebrafish models for translational neuroscience research: from tank to bedside. Trends in neurosciences 37, 264-278. 
Stewart, A.M., Gerlai, R., Kalueff, A.V., 2015a. Developing highER-throughput zebrafish screens for in-vivo CNS drug discovery. Frontiers in behavioral neuroscience 9, 14.

Stewart, A.M., Grossman, L., Collier, A.D., Echevarria, D.J., Kalueff, A.V., $2015 b$. Anxiogenic-like effects of chronic nicotine exposure in zebrafish. Pharmacology, biochemistry, and behavior $139 \mathrm{Pt} \mathrm{B}, 112-120$.

Stratton, K.R., Howe, C.J., Battaglia, F.C., Institute of Medicine (U.S.). Division of Biobehavioral Sciences and Mental Disorders. Committee to Study Fetal Alcohol Syndrome, National Institute on Alcohol Abuse and Alcoholism (U.S.) 1996. Fetal alcohol syndrome: diagnosis, epidemiology, prevention, and treatment. Washington DC: National Academy Press

Streissguth, A.P., Herman, C.S., Smith, D.W., 1978. Intelligence, behavior, and dysmorphogenesis in the fetal alcohol syndrome: a report on 20 patients. The Journal of pediatrics 92, 363-367.

Sugathan, A., Biagioli, M., Golzio, C., Erdin, S., Blumenthal, I., Manavalan, P., et al., 2014. CHD8 regulates neurodevelopmental pathways associated with autism spectrum disorder in neural progenitors. Proceedings of the National Academy of Sciences of the United States of America 111, E4468-4477.

Suriyampola, P.S., Shelton, D.S., Shukla, R., Roy, T., Bhat, A., Martins, E.P., 2016. Zebrafish Social Behavior in the Wild. Zebrafish 13, 1-8.

Tabassum, N., Tai, H., Jung, D.W., Williams, D.R., 2015. Fishing for Nature's Hits: Establishment of the Zebrafish as a Model for Screening Antidiabetic Natural 
Products. Evidence-based complementary and alternative medicine: eCAM 2015, 287847.

Tay, T.L., Ronneberger, O., Ryu, S., Nitschke, R., Driever, W., 2011. Comprehensive catecholaminergic projectome analysis reveals single-neuron integration of zebrafish ascending and descending dopaminergic systems. Nature communications 2,171 .

Teles, M.C., Dahlbom, S.J., Winberg, S., Oliveira, R.F., 2013. Social modulation of brain monoamine levels in zebrafish. Behavioral brain research 253, 17-24.

Teles, M.C., Oliveira, R.F., 2016. Quantifying Aggressive Behavior in Zebrafish. Methods in molecular biology (Clifton, N.J.) 1451, 293-305.

Tellez-Zenteno, J.F., Patten, S.B., Jette, N., Williams, J., Wiebe, S., 2007. Psychiatric comorbidity in epilepsy: a population-based analysis. Epilepsia 48, 2336-2344.

Torres-Hernandez, B.A., Colon, L.R., Rosa-Falero, C., Torrado, A., Miscalichi, N., Ortiz, J.G., et al., 2016. Reversal of pentylenetetrazole-altered swimming and neural activity-regulated gene expression in zebrafish larvae by valproic acid and valerian extract. Psychopharmacology 233, 2533-2547.

Torres-Hernandez, B.A., Del Valle-Mojica, L.M., Ortiz, J.G., 2015. Valerenic acid and Valeriana officinalis extracts delay onset of Pentylenetetrazole (PTZ)-Induced seizures in adult Danio rerio (Zebrafish). BMC complementary and alternative medicine 15, 228.

Tropepe, V., Sive, H.L., 2003. Can zebrafish be used as a model to study the neurodevelopmental causes of autism? Genes, brain, and behavior 2, 268-281. 
Turner, K.J., Bracewell, T.G., Hawkins, T.A., 2014. Anatomical dissection of zebrafish brain development. Methods in molecular biology (Clifton, N.J.) 1082, 197-214. Voineagu, I., Wang, X., Johnston, P., Lowe, J.K., Tian, Y., Horvath, S., Mill, J., Cantor, R.M., Blencowe, B.J., Geschwind, D.H., 2011. Transcriptomic analysis of autistic brain reveals convergent molecular pathology. Nature 474, 380-384.

Way, G.P., Ruhl, N., Snekser, J.L., Kiesel, A.L., McRobert, S.P., 2015. A comparison of methodologies to test aggression in zebrafish. Zebrafish 12, 144-151.

Willsey, A.J., Sanders, S.J., Li, M., Dong, S., Tebbenkamp, A.T., Muhle, R.A., Reilly, S.K., Lin, L., Fertuzinhos, S., Miller, J.A., Murtha, M.T., Bichsel, C., Niu, W., Cotney, J., Ercan-Sencicek, A.G., Gockley, J., Gupta, A.R., Han, W., He, X., Hoffman, E.J., Klei, L., Lei, J., Liu, W., Liu, L., Lu, C., Xu, X., Zhu, Y., Mane, S.M., Lein, E.S., Wei, L., Noonan, J.P., Roeder, K., Devlin, B., Sestan, N., State, M.W., 2013. Coexpression networks implicate human midfetal deep cortical projection neurons in the pathogenesis of autism. Cell 155, 997-1007.

Wilson, S.W., Ross, L.S., Parrett, T., Easter, S.S., Jr., 1990. The development of a simple scaffold of axon tracts in the brain of the embryonic zebrafish, Brachydanio rerio. Development (Cambridge, England) 108, 121-145.

Wolman, M.A., Jain, R.A., Liss, L., Granato, M., 2011. Chemical modulation of memory formation in larval zebrafish. Proceedings of the National Academy of Sciences of the United States of America 108, 15468-15473.

Wright, G.J., Washbourne, P., 2011. Neurexins, neuroligins and LRRTMs: synaptic adhesion getting fishy. Journal of neurochemistry 117, 765-778. 
Wright, D., Rimmer, L.B., Pritchard, V.L., Krause, J., Butlin, R.K., 2003. Inter and intrapopulation variation in shoaling and boldness in the zebrafish (Danio rerio). Die Naturwissenschaften 90, 374-377.

Zellner, D., Padnos, B., Hunter, D.L., MacPhail, R.C., Padilla, S., 2011. Rearing conditions differentially affect the locomotor behavior of larval zebrafish, but not their response to valproate-induced developmental neurotoxicity. Neurotoxicology and teratology 33, 674-679.

Zerucha, T., Stuhmer, T., Hatch, G., Park, B.K., Long, Q., Yu, G., Gambarotta, A., Schultz, J.R., Rubenstein, J.L., Ekker, M., 2000. A highly conserved enhancer in the Dlx5/Dlx6 intergenic region is the site of cross-regulatory interactions between Dlx genes in the embryonic forebrain. The Journal of neuroscience: the official journal of the Society for Neuroscience 20, 709-721.

Zhdanova, I.V., Wang, S.Y., Leclair, O.U., Danilova, N.P., 2001. Melatonin promotes sleep-like state in zebrafish. Brain research 903, 263-268.

\section{FIGURE LEGENDS}

Figure 1. Group vs Individual testing. Adult zebrafish prefer to move in groups (shoaling). In these experiments, animals were tested in groups (3 fish/group) and retested as individuals half hour later. One hour after being regrouped, there is still considerable dispersion in the time spent in the light area.

Figure 2. Anxiolytic Effects of aqueous Citrus auratium extract on zebrafish in the lightdark preference task. (Del Valle-Mojica and Ortiz, 2012). Zebrafish were tested in the 
light-dark test before (naïve) and after a one-hour long exposure to $20 \mathrm{mg} / \mathrm{mL}$ extract for one hour.

Figure 3. Behavioral and neurochemical changes in adult zebrafish induced by embryonic alcohol exposure. Panel A shows the reduction of distance elicited by the presentation of moving (animated) images of conspecifics (zebrafish). Mean \pm S.E.M. are shown The larger negative values represent more robust reduction. Note that control (alcohol unexposed) zebrafish reduced their distance to the stimulus by $16 \mathrm{~cm}$ on average, but the response of zebrafish exposed previously to $1 \%$ alcohol is statistically indistinguishable from chance (i.e. no response to conspecific images). Also note the quasi-linear dose response relationship between behavioral response and alcohol concentration. Panel B shows the amount of dopamine relative to total protein weight as measured by HPLC from whole brain extract. Mean \pm S.E.M. are shown. The pairs of bars represent behavioral performance of fish exposed to alcohol at different concentrations (representing $\mathrm{Vol} / \mathrm{Vol} \%$ ) of alcohol during embryonic development. The first bar in each pair shows the relative dopamine amount before conspecific images are shown. The second bar shows the relative dopamine amount right after the conspecific images were shown. Note the robust increase of dopamine in response to the images in control fish. Also note the complete absence of this increase in alcohol exposed fish. Modified from Fernandes and Gerlai (2009) and Fernandes et al., 2015a) 